\title{
Derivative-free optimization: a review of algorithms and comparison of software implementations
}

\author{
Luis Miguel Rios • Nikolaos V. Sahinidis
}

Received: 20 December 2011 / Accepted: 23 June 2012 / Published online: 12 July 2012

(C) Springer Science+Business Media, LLC. 2012

\begin{abstract}
This paper addresses the solution of bound-constrained optimization problems using algorithms that require only the availability of objective function values but no derivative information. We refer to these algorithms as derivative-free algorithms. Fueled by a growing number of applications in science and engineering, the development of derivativefree optimization algorithms has long been studied, and it has found renewed interest in recent time. Along with many derivative-free algorithms, many software implementations have also appeared. The paper presents a review of derivative-free algorithms, followed by a systematic comparison of 22 related implementations using a test set of 502 problems. The test bed includes convex and nonconvex problems, smooth as well as nonsmooth problems. The algorithms were tested under the same conditions and ranked under several criteria, including their ability to find near-global solutions for nonconvex problems, improve a given starting point, and refine a near-optimal solution. A total of 112,448 problem instances were solved. We find that the ability of all these solvers to obtain good solutions diminishes with increasing problem size. For the problems used in this study, TOMLAB / MULTIMIN, TOMLAB/GLCCLUSTER, MCS and TOMLAB/LGO are better, on average, than other derivative-free solvers in terms of solution quality within 2,500 function evaluations. These global solvers outperform local solvers even for convex problems. Finally, TOMLAB / OQNLP, NEWUOA, and TOMLAB / MULTIMIN show superior performance in terms of refining a nearoptimal solution.
\end{abstract}

Keywords Derivative-free algorithms $\cdot$ Direct search methods $\cdot$ Surrogate models

Electronic supplementary material The online version of this article (doi:10.1007/s10898-012-9951-y) contains supplementary material, which is available to authorized users.

L. M. Rios · N. V. Sahinidis $(\bowtie)$

Department of Chemical Engineering,

Carnegie Mellon University, Pittsburgh, PA 15213, USA

e-mail: sahinidis@cmu.edu

L. M. Rios

e-mail:1mrios@gmail.com 


\section{Introduction}

The problem addressed in this paper is the optimization of a deterministic function $f$ : $\mathbb{R}^{n} \rightarrow \mathbb{R}$ over a domain of interest that possibly includes lower and upper bounds on the problem variables. We assume that the derivatives of $f$ are neither symbolically nor numerically available, and that bounds, such as Lipschitz constants, for the derivatives of $f$ are also unavailable.

The problem is of interest when derivative information is unavailable, unreliable, or impractical to obtain, for instance when $f$ is expensive to evaluate or somewhat noisy, which renders most methods based on finite differences of little or no use $[79,140]$. We refer to this problem as derivative-free optimization. We further refer to any algorithm applied to this problem as a derivative-free algorithm, even if the algorithm involves the computation of derivatives for functions other than $f$.

Derivative-free optimization is an area of long history and current rapid growth, fueled by a growing number of applications that range from science problems $[4,42,52,143]$ to medical problems $[90,103]$ to engineering design and facility location problems $[2,10,15,48,49,54$, $57,91,92,98]$.

The development of derivative-free algorithms dates back to the works of Spendley et al. [132] and Nelder and Mead [99] with their simplex-based algorithms. Recent works on the subject have led to significant progress by providing convergence proofs $[5,9,31,34,76,80$, $85,88,134]$, incorporating the use of surrogate models [22,24,127,131], and offering the first textbook that is exclusively devoted to this topic [35]. Concurrent with the development of algorithms, software implementations for this class of optimization problems have resulted in a wealth of software packages, including BOBYQA [115], CMA-ES [55], four COLINY solvers [124], DFO [125], glcCluster [60, pp. 109-111], HOPSPACK [108], IMFIL [74], LGO [105], MCS [101], multimin [60, pp. 148-151], NEWUOA [114], NOMAD [6], OQNLP [62], PSWARM [137,138], SID-PSM [40,41], and SNOBFIT [66].

Mongeau et al. [96] performed a comparison of derivative-free solvers in 1998 by considering six solvers over a set of eleven test problems. Since the time of that comparison, the number of available derivative-free optimization solvers has more than quadrupled. Other comparisons, such as $[11,33,38,48,53,65,67,97,138]$, are restricted to a few solvers related to the algorithms proposed in these papers. In addition, these comparisons consider small sets of test problems. There is currently no systematic comparison of the existing implementations of derivative-free optimization algorithms on a large collection of test problems. The primary purpose of the present paper is to provide such a comparison, aiming at addressing the following questions:

- What is the quality of solutions obtained by current solvers for a given limit on the number of allowable function evaluations? Does quality drop significantly as problem size increases?

- Which solver is more likely to obtain global or near-global solutions for nonconvex problems?

- Is there a subset of existing solvers that would suffice to solve a large fraction of problems when all solvers are independently applied to all problems of interest? Conversely, are there problems that can be solved by only one or a few solvers?

- Given a starting near-optimal solution, which solver reaches the solution fastest?

Before addressing these questions computationally, the paper begins by presenting a review of the underlying theory and motivational ideas of the algorithms. We classify algorithms developed for this problem as direct and model-based. Direct algorithms determine 
search directions by computing values of the function $f$ directly, whereas model-based algorithms construct and utilize a surrogate model of $f$ to guide the search process. We further classify algorithms as local or global, with the latter having the ability to refine the search domain arbitrarily. Finally, we classify algorithms as stochastic or deterministic, depending upon whether they require random search steps or not.

Readers familiar with the subject matter of this paper may have noticed that there exists literature that reserves the term derivative-free algorithms only for what we refer to as modelbased algorithms in the current paper. In addition, what we refer to as derivative-free optimization is often also referred to as optimization over black boxes. The literature on these terms is often inconsistent and confusing (cf. [79] and discussion therein).

Local search algorithms are reviewed in Sect. 2. The presentation includes direct and model-based strategies. Global search algorithms are discussed in Sect. 3, including deterministic as well as stochastic approaches. Section 4 provides a brief historical overview and overall assessment of the algorithmic state-of-the-art in this area. Leading software implementations of derivative-free algorithms are discussed in Sect. 5. The discussion includes software characteristics, requirements, and types of problems handled. Extensive computational experience with these codes is presented in Sects. 6 and 7. A total of 502 test problems were used, including 78 smooth convex, 161 nonsmooth convex, 245 smooth nonconvex, and 18 nonsmooth nonconvex problems. These problems were used to test 22 solvers using the same starting points and bounding boxes. During the course of the computational work for this paper, we made test problems and results available to the software developers and asked them to provide us with a set of options of their choice. Many of them responded and even decided to change the default settings in their software thereafter. In an effort to encourage developers to revise and improve their software, we shared our computational results with them over several rounds. Between each round, several developers provided improved versions of their implementations. At the end of this process, we tested their software against an additional collection of problems that had not been seen by developers in order to confirm that the process had not led to overtraining of the software on our test problem collection. Conclusions from this entire study are drawn in Sect. 8.

\section{Local search methods}

\subsection{Direct local search methods}

Hooke and Jeeves [64] describe direct search as the sequential examination of trial solutions generated by a certain strategy. Classical direct search methods did not come with proofs of termination or convergence to stationary points. However, recent papers starting with $[134,135]$ have proved convergence to stationary points, among other properties. These old methods remain popular due to their simplicity, flexibility, and reliability. We next describe specific direct search methods that are local in nature.

\subsubsection{Nelder-Mead simplex algorithm}

The Nelder-Mead algorithm introduced in [99] starts with a set of points that form a simplex. In each iteration, the objective function values at the corner points of the simplex determine the worst corner point. The algorithm attempts to replace the worst point by introducing a new vertex in a way that results in a new simplex. Candidate replacement points are obtained by transforming the worst vertex through a number of operations about the centroid of the 
current simplex: reflection, expansion, inside and outside contractions. McKinnon [94] has established analytically that convergence of the Nelder-Mead algorithm can occur to a point where the gradient of the objective function is nonzero, even when the function is convex and twice continuously differentiable. To prevent stagnation, Kelley [75] proposed to enforce a sufficient decrease condition determined by an approximation of the gradient. If stagnation is observed, the algorithm restarts from a different simplex. Tseng [136] proposed a globally convergent simplex-based search method that considers an expanded set of candidate replacement points. Other modifications of the Nelder-Mead algorithm are presented in [35].

\subsubsection{Generalized pattern search (GPS) and generating set search (GSS) methods}

Torczon [135] introduced generalized pattern search methods (GPS) for unconstrained optimization. GPS generalizes direct search methods including the Hooke and Jeeves [64] algorithm. At the beginning of a new iteration, GPS searches by exploratory moves. The current iterate defines a set of points that form a pattern, determined by a step and a generating matrix that spans $\mathbb{R}^{n}$.

Further generalizing GPS, Kolda et al. [79] coined the term GSS in order to describe, unify, and analyze direct search methods, including algorithms that apply to constrained problems. Each iteration $k$ of GSS methods consists of two basic steps. The search step is performed first over a finite set of search directions $\mathcal{H}_{k}$ generated by some, possibly heuristic, strategy that aims to improve the current iterate but may not guarantee convergence. If the search step fails to produce a better point, GSS methods continue with the poll step, which is associated with a generating set $\mathcal{G}_{k}$ that spans positively $\mathbb{R}^{n}$. Generating sets are usually positive bases, with a cardinality between $n+1$ to $2 n$. Assuming $f$ is smooth, a generating set contains at least one descent direction of $f$ at a non-stationary point in the domain of definition of $f$.

Given $\mathcal{G}=\left\{d^{(1)}, \ldots, d^{(p)}\right\}$ with $p \geq n+1$ and $d^{(i)} \in \mathbb{R}^{n}$, the function $f$ is evaluated at a set of trial points $\mathcal{P}_{k}=\left\{x_{k}+\triangle_{k} d: d \in \mathcal{G}_{k}\right\}$, where $\triangle_{k}$ is the step length. An iteration is successful if there exists $y \in \mathcal{P}_{k}$ such that $f(y)<f\left(x_{k}\right)-\rho\left(\triangle_{k}\right)$, where $\rho$ is a forcing function. The opportunistic poll strategy proceeds to the next iteration upon finding a point $y$, while the complete poll strategy evaluates all points in $\mathcal{P}_{k}$ and assigns $y=\arg \min _{x \in \mathcal{P}_{k}} f(x)$. Successful iterations update the iterate $x_{k+1}$ to $y$ and possibly increase the step length $\triangle_{k+1}$ to $\phi_{k} \triangle_{k}$, with $\phi_{k} \geq 1$. Unsuccessful iterations maintain the same iterate, i.e., $x_{k+1}=x_{k}$, and reduce the step length $\triangle_{k+1}$ to $\theta_{k} \triangle_{k}$, with $0<\theta_{k}<1$. The forcing function $\rho$ is included in order to impose a sufficient decrease condition and is required to be a continuous decreasing function with $\lim _{t \rightarrow 0} \rho(t) / t=0$. Alternatively, the set $\mathcal{P}_{k}$ can be generated using integer lattices [135]. In the latter case, the iteration is successful if a point $y$ satisfies simple decrease, i.e., $f(y)<f\left(x_{k}\right)$.

Lewis and Torczon [83] extended pattern search methods to problems with bound constraints by including axis directions in the set of poll directions. Lewis and Torczon [84] further extended pattern search methods to linearly constrained problems by forcing the generating set to span a tangent cone $\mathcal{T}_{\Omega}\left(x_{k}, \epsilon\right)$, which restricts the tangent vectors to satisfy all constraints within an $\epsilon$-neighborhood from $x_{k}$.

Under mild conditions, [135] showed convergence of GSS methods to stationary points, which, could be local minima, local maxima, or even saddle points.

Mesh adaptive direct search (MADS) methods The MADS methods (Audet and Dennis [11]) modified the poll step of GPS algorithms to consider a variable set of poll directions whose union across all iterations is asymptotically dense in $\mathbb{R}^{n}$. MADS generates the poll points using two parameters: a poll size parameter, which restricts the region from which points can 
be selected, and a mesh size parameter, which defines a grid inside the region limited by the poll size parameter. MADS incorporates dynamic ordering, giving precedence to previously successful poll directions. Audet and Dennis [11] first proposed random generation of poll directions for each iteration, and Audet et al. [8] proposed a deterministic way for generating orthogonal directions.

Abramson and Audet [5] showed convergence of the MADS method to second-order stationary points under the assumption that $f$ is continuously differentiable with Lipschitz derivatives near the limit point. Under additional assumptions ( $f$ twice strictly differentiable near the limit point), MADS was shown to converge to a local minimizer with probability 1. A number of problems for which GPS stagnates and MADS converges to an optimal solution are presented in [11]. Some examples were presented in [5] that show how GPS methods stall at saddle points, while MADS escapes and converges to a local minimum.

MADS handles general constraints by the extreme barrier approach [11], which rejects infeasible trial points from consideration, or by the progressive barrier approach [12], which allows infeasible trial points within a decreasing infeasibility threshold.

Pattern search methods using simplex gradients Custódio and Vicente [40] proposed to enhance the poll step by giving preference to directions that are closest to the negative of the simplex gradient. Simplex gradients are an approximation to the real gradient and are calculated out of a simplex defined by previously evaluated points.

\subsection{Local model-based search algorithms}

The availability of a high-fidelity surrogate model permits one to exploit its underlying properties to guide the search in a intelligent way. Properties such as the gradient and higher order derivative information, as well as the probability distribution function of the surrogate model are used. Since a high-fidelity surrogate model is typically unavailable for a given problem, these methods start by sampling the search space and building an initial surrogate model. The methods then proceed iteratively to optimize the surrogate model, evaluate the solution point, and update the surrogate model.

\subsubsection{Trust-region methods}

Trust-region methods use a surrogate model that is usually smooth, easy to evaluate, and presumed to be accurate in a neighborhood (trust region) about the current iterate. Powell [109] proposed to use a linear model of the objective within a trust-region method. The algorithm considered a monotonically decreasing radius parameter and included iterations that maintained geometric conditions of the interpolation points. Linear models are practical since they only require $\mathcal{O}(n)$ interpolation points, albeit at the cost of not capturing the curvature of the underlying function. Powell [112] and Conn et al. [31,32] proposed to use a quadratic model of the form:

$$
q_{k}\left(x_{k}+s\right)=f\left(x_{k}\right)+\left\langle g_{k}, s\right\rangle+\frac{1}{2}\left\langle s, H_{k} s\right\rangle
$$

where, at iteration $k, x_{k}$ is the current iterate, $g_{k} \in \mathbb{R}^{n}$, and $H_{k}$ is a symmetric matrix of dimension $n$. Rather than using derivative information, $g_{k}$ and $H_{k}$ are estimated by requiring $q_{k}$ to interpolate a set $Y$ of sample points: $q_{k}\left(x^{(i)}\right)=f\left(x^{(i)}\right)$ for $i=1, \ldots, p$. Unless conditions are imposed on the elements of $g_{k}$ and $H_{k}$, at least $(n+1)(n+2) / 2$ points are needed to determine $g_{k}$ and $H_{k}$ uniquely. Let $x^{*}$ denote a minimizer of $q_{k}$ within the trust 
region and define the ratio $\rho_{k}=\left(f\left(x_{k}\right)-f\left(x^{*}\right)\right) /\left(q_{k}\left(x_{k}\right)-q_{k}\left(x^{*}\right)\right)$. If $\rho_{k}$ is greater than a user-defined threshold, $x^{*}$ replaces a point in $Y$ and the trust region is increased. Otherwise, if the geometry of the set $Y$ is adequate, the trust-region radius is reduced, while, if the geometry is not adequate, a point in the set is replaced by another that improves the poisedness of the set. The algorithm terminates when the trust-region radius drops below a given tolerance.

Powell [113] proposed an algorithm that uses a quadratic model relying on fewer than $(n+1)(n+2) / 2$ interpolation points. The remaining degrees of freedom in the interpolation are determined by minimizing the change to the Hessian of the surrogate model between two consecutive iterations.

\subsubsection{Implicit filtering}

In addition to, or instead of developing a surrogate of $f$, one may develop a surrogate of the gradient of $f$ and use it to expedite the search. Implicit filtering [50,142] uses an approximation of the gradient to guide the search, resembling the steepest descent method when the gradient is known. The approximation of the gradient at an iterate is based on forward or centered differences, and the difference increment varies as the optimization progresses. Forward differences require $n$ function evaluations, whereas centered difference gradients require $2 n$ function evaluations over the set $\left\{x \pm s e_{i}: i=1, \ldots, n\right\}$, where $x$ is the current iterate, $s$ is the scale of the stencil, and $e_{i}$ are coordinate unit vectors. As these points are distributed around the iterate, they produce approximations less sensitive to noise than forward differences [50]. A line search is then performed along the direction of the approximate gradient. The candidate point is required to satisfy a minimum decrease condition of the form $f\left(x-\delta \nabla_{s} f(x)\right)-f(x)<-\alpha \delta\left\|\nabla_{s} f(x)\right\|^{2}$, where $\delta$ is the step and $\alpha$ is a parameter. The algorithm continues until no point satisfies the minimum decrease condition, at which point the scale $s$ is decreased. The implicit filtering algorithm terminates when the approximate gradient is less than a certain tolerance proportional to $s$.

\section{Global search algorithms}

\subsection{Deterministic global search algorithms}

\subsubsection{Lipschitzian-based partitioning techniques}

Lipschitzian-based methods construct and optimize a function that underestimates the original one. By constructing this underestimator in a piecewise fashion, these methods provide possibilities for global, as opposed to only local, optimization of the original problem. Let $L>0$ denote a Lipschitz constant of $f$. Then $|f(a)-f(b)| \leq L\|a-b\|$ for all $a, b$ in the domain of $f$. Assuming $L$ is known, Shubert [129] proposed an algorithm for boundconstrained problems. This algorithm evaluates the extreme points of the search space and constructs linear underestimators by means of the Lipschitz constant. The algorithm then proceeds to evaluate the minimum point of the underestimator and construct a piecewise underestimator by partitioning the search space.

A straightforward implementation of Shubert's algorithm for derivative-free optimization problems has two major drawbacks: the Lipschitz constant is unknown and the number of function evaluations increases exponentially, as the number of extreme points of an $n$-dimensional hypercube is $2^{n}$. The DIRECT algorithm and branch-and-bound search are two possible approaches to address these challenges. 
The DIRECT algorithm Jones et al. [72] proposed the DIRECT algorithm (DIvide a hyperRECTangle), with two main ideas to extend Shubert's algorithm to derivative-free optimization problems. First, function values are computed only at the center of an interval, instead of all extreme points. By subdividing intervals into thirds, one of the resulting partition elements inherits the center of the initial interval, where the objective function value is already known. The second main idea of the DIRECT algorithm is to select from among current hyperrectangles one that (a) has the lowest objective function value for intervals of similar size and (b) is associate with a large potential rate of decrease of the objective function value. The amount of potential decrease in the current objective function value represents a setable parameter in this algorithm and can be used to balance local and global search; larger values ensure that the algorithm is not local in its orientation.

In the absence of a Lipschitz constant, the DIRECT algorithm terminates once the number of iterations reaches a predetermined limit. Under mild conditions, Finkel and Kelley [47] proved that the sequence of best points generated by the algorithm converges to a KKT point. Convergence was also established for general constrained problems, using a barrier approach.

Branch-and-bound $(B B)$ search BB sequentially partitions the search space, and determines lower and upper bounds for the optimum. Partition elements that are inferior are eliminated in the course of the search. Let $\Omega=\left[x_{l}, x_{u}\right]$ be the region of interest and let $x_{\Omega}^{*} \in \Omega$ be a global minimizer of $f$ in $\Omega$. The availability of a Lipschitz constant $L$ along with a set of sample points $\Lambda=\left\{x^{(i)}, i=1, \ldots, p\right\} \subset \Omega$ provides lower and upper bounds:

$$
\max _{i=1, \ldots, p}\left\{f\left(x^{(i)}\right)-L \delta_{i}\right\}=\underline{f}_{\Omega} \leq f\left(x_{\Omega}^{*}\right) \leq \bar{f}_{\Omega}=\min _{i=1, \ldots, p} f\left(x^{(i)}\right),
$$

where $\delta_{i}$ is a function of the distance of $x^{(i)}$ from the vertices of $\left[x_{l}, x_{u}\right]$. The Lipschitz constant $L$ is unknown but a lower bound $\underline{L}$ can be estimated from the sampled objective function values:

$$
\underline{L}=\max _{i, j} \frac{\left|f\left(x^{(i)}\right)-f\left(x^{(j)}\right)\right|}{\left\|x^{(i)}-x^{(j)}\right\|} \leq L, \quad i, j=1, \ldots, p, \quad i \neq j .
$$

Due to the difficulty of obtaining deterministic upper bounds for $L$, statistical bounds relying on extreme order statistics were proposed in [106]. This approach assumes samples are generated randomly from $\Omega$ and their corresponding objective function values are random variables. Subset-specific estimates of $L$ can be significantly smaller than the global $L$, thus providing sharper lower bounds for the objective function as BB iterations proceed.

\subsubsection{Multilevel coordinate search (MCS)}

Like the DIRECT algorithm, MCS [65] partitions the search space into boxes with an evaluated base point. Unlike the DIRECT algorithm, MCS allows base points anywhere in the corresponding boxes. Boxes are divided with respect to a single coordinate. The global-local search that is conducted is balanced by a multilevel approach, according to which each box is assigned a level $s$ that is an increasing function of the number of times the box has been processed. Boxes with level $s=s_{\max }$ are considered too small to be further split.

At each iteration, MCS selects boxes with the lowest objective value for each level value and marks them as candidates for splitting. Let $n_{j}$ be the number of splits in coordinate $j$ during the course of the algorithm. If $s>2 n\left(\min n_{j}+1\right)$, open boxes are considered for splitting by rank, which prevents having unexplored coordinates for boxes with high $s$ values; 
in this case, the splitting index $k$ is chosen such that $n_{k}=\min n_{j}$. Otherwise, open boxes are considered for splitting by expected gain, which selects the splitting index and coordinate value by optimizing a local separable quadratic model using previously evaluated points. MCS with local search performs local searches from boxes with level $s_{\max }$, provided that the corresponding base points are not near previously investigated points. As $s_{\max }$ approaches infinity, the base points of MCS form a dense subset of the search space and MCS converges to a global minimum [65].

\subsection{Global model-based search algorithms}

Similarly to local model-based algorithms described in Sect. 2.2, global model-based approaches optimize a high-fidelity surrogate model, which is evaluated and updated to guide the optimization of the real model. In this context, the surrogate model is developed for the entire search space or subsets that are dynamically refined through partitioning.

\subsubsection{Response surface methods (RSMs)}

These methods approximate an unknown function $f$ by a response surface (or metamodel) $\hat{f}[16]$. Any mismatch between $f$ and $\hat{f}$ is assumed to be caused by model error and not because of noise in experimental measurements.

Response surfaces may be non-interpolating or interpolating [70]. The former are obtained by minimizing the sum of square deviations between $f$ and $\hat{f}$ at a number of points, where measurements of $f$ have been obtained. The latter produce functions that pass through the sampled responses. A common choice for non-interpolating surfaces are low-order polynomials, the parameters of which are estimated by least squares regression on experimental designs. Interpolating methods include kriging and radial basis functions. Independent of the functions used, the quality of the predictor depends on selecting an appropriate sampling technique [14].

The interpolating predictor at point $x$ is of the form:

$$
\hat{f}(x)=\sum_{i=1}^{m} \alpha_{i} f_{i}(x)+\sum_{i=1}^{p} \beta_{i} \varphi\left(x-x^{(i)}\right),
$$

where $f_{i}$ are polynomial functions, $\alpha_{i}$ and $\beta_{i}$ are unknown coefficients to be estimated, $\varphi$ is a basis function, and $x^{(i)} \in \mathbb{R}^{n}, i=1, \ldots, p$, are sample points. Basis functions include linear, cubic, thin plate splines, multiquadratic, and kriging. These are discussed below in more detail.

Kriging Originally used for mining exploration models, kriging [93] models a deterministic response as the realization of a stochastic process by means of a kriging basis function. The interpolating model that uses a kriging basis function is often referred to as a Design and Analysis of Computer Experiments (DACE) stochastic model [122]:

$$
\begin{array}{r}
\hat{f}(x)=\mu+\sum_{i=1}^{p} b_{i} \exp \left[-\sum_{h=1}^{n} \theta_{h}\left|x_{h}-x_{h}^{(i)}\right|^{p_{h}}\right], \\
\theta_{h} \geq 0, \quad p_{h} \in[0,2], h=1, \ldots, n .
\end{array}
$$

Assuming $\hat{f}$ is a random variable with known realizations $\hat{f}\left(x^{(i)}\right), i=1, \ldots, p$, the parameters $\mu, b_{i}, \theta_{h}$ and $p_{h}$ are estimated by maximizing the likelihood of the observed 
realizations. The parameters are dependent on sample point information but independent of the candidate point $x$. Nearby points are assumed to have highly correlated function values, thus generating a continuous interpolating model. The weights $\theta_{h}$ and $p_{h}$ account for the importance and the smoothness of the corresponding variables. The predictor $\hat{f}(x)$ is then minimized over the entire domain.

Efficient global optimization (EGO) The EGO algorithm [73,126] starts by performing a space-filling experimental design. Maximum likelihood estimators for the DACE model are calculated and the model is then tested for consistency and accuracy. A branch-andbound algorithm is used to optimize the expected improvement, $E[I(x)]$, at the point $x$. This expected improvement is defined as: $\mathrm{E}[I(x)]=\mathrm{E}\left[\max \left(f_{\min }-\hat{f}(x), 0\right)\right]$, where $f_{\min }$ is the best objective value known and $\hat{f}$ is assumed to follow a normal distribution with mean and standard deviation equal to the DACE predicted values. Although the expected improvement function can be reduced to a closed-form expression [73], it can be highly multimodal.

Radial basis functions Radial basis functions approximate $f$ by considering an interpolating model based on radial functions. Powell [111] introduced radial basis functions to derivative-free optimization.

Given a set of sample points, Gutmann [53] proposed to find a new point $\bar{x}$ such that the updated interpolant predictor $\hat{f}$ satisfies $\hat{f}(\bar{x})=T$ for a target value $T$. Assuming that smooth functions are more likely than "bumpy" functions, $\bar{x}$ is chosen to minimize a measure of "bumpiness" of $\hat{f}$. This approach is similar to maximizing the probability of improvement [70], where $\underline{x}$ is chosen to maximize the probability: Prob $=\Phi[(T-\hat{f}(\underline{x})) / s(\underline{x})]$, where $\Phi$ is the normal cumulative distribution function and $s(\underline{x})$ is the standard deviation predictor.

Various strategies that rely on radial basis functions have been proposed and analyzed $[63,103,118]$, as well as extended to constrained optimization [117]. The term "RBF methods" will be used in later sections to refer to global optimization algorithms that minimize the radial-basis-functions-based interpolant directly or minimize a measure of bumpiness.

Sequential design for optimization (SDO) Assuming that $\hat{f}(x)$ is a random variable with standard deviation predictor $s(x)$, the SDO algorithm [36] proposes the minimization of the statistical lower bound of the function $\hat{f}\left(x^{*}\right)-\tau s\left(x^{*}\right)$ for some $\tau \geq 0$.

\subsubsection{Surrogate management framework $(S M F)$}

Booker et al. [23] proposed a pattern search method that utilizes a surrogate model. SMF involves a search step that uses points generated by the surrogate model in order to produce potentially optimal points as well as improve the accuracy of the surrogate model. The search step alternates between evaluating candidate solution points and calibrating the surrogate model until no further improvement occurs, at which point the algorithm switches to the poll step.

\subsubsection{Optimization by branch-and-fit}

Huyer and Neumaier [66] proposed an algorithm that combines surrogate models and randomization. Quadratic models are fitted around the incumbent, whereas linear models are fitted around all other evaluated points. Candidate points for evaluation are obtained by optimizing these models. Random points are generated when the number of points at hand is 
insufficient to fit the models. Additional points from unexplored areas are selected for evaluation. The user provides a resolution vector that confines the search to its multiples, thereby defining a grid of candidate points. Smaller resolution vectors result in grids with more points.

\subsection{Stochastic global search algorithms}

This section presents approaches that rely on critical non-deterministic algorithmic steps. Some of these algorithms occasionally allow intermediate moves to lesser quality points than the solution currently at hand. The literature on stochastic algorithms is very extensive, especially on the applications side, since their implementation is rather straightforward compared to deterministic algorithms.

\subsubsection{Hit-and-run algorithms}

Proposed independently by Boneh and Golan [21] and Smith [130], each iteration of hitand-run algorithms compares the current iterate $x$ with a randomly generated candidate. The current iterate is updated only if the candidate is an improving point. The generation of candidates is based on two random components. A direction $d$ is generated using a uniform distribution over the unit sphere. For the given $d$, a step $s$ is generated from a uniform distribution over the set of steps $S$ in a way that $x+d s$ is feasible. Bélisle et al. [17] generalized hit-and-run algorithms by allowing arbitrary distributions to generate both the direction $d$ and step $s$, and proved convergence to a global optimum under mild conditions for continuous optimization problems.

\subsubsection{Simulated annealing}

At iteration $k$, simulated annealing generates a new trial point $\hat{x}$ that is compared to the incumbent $x^{k}$ and accepted with a probability function [95]:

$$
\mathrm{P}\left(\hat{x} \mid x_{k}\right)= \begin{cases}\exp \left[-\frac{f(\hat{x})-f\left(x_{k}\right)}{T_{k}}\right] & \text { if } f(\hat{x})>f\left(x_{k}\right) \\ 1 & \text { if } f(\hat{x}) \leq f\left(x_{k}\right) .\end{cases}
$$

As a result, unlike hit-and-run algorithms, simulated annealing allows moves to points with objective function values worse than the incumbent. The probability $\mathrm{P}$ depends on the "temperature" parameter $T_{k}$; the sequence $\left\{T_{k}\right\}$ is referred to as the cooling schedule. Cooling schedules are decreasing sequences that converge to 0 sufficiently slow to permit the algorithm to escape from local optima.

Initially proposed to handle combinatorial optimization problems [78], the algorithm was later extended to continuous problems [17]. Asymptotic convergence results to a global optimum have been presented [1] but there is no guarantee that a good solution will be obtained in a finite number of iterations [121]. Interesting finite-time performance aspects are discussed in $[27,104]$.

\subsubsection{Genetic algorithms}

Genetic algorithms, often referred to as evolutionary algorithms, were introduced by Holland [58] and resemble natural selection and reproduction processes governed by rules that assure the survival of the fittest in large populations. Individuals (points) are associated with identity genes that define a fitness measure (objective function value). A set of individuals 
form a population, which adapts and mutates following probabilistic rules that utilize the fitness function. Bethke [18] extended genetic algorithms to continuous problems by representing continuous variables by an approximate binary decomposition. Liepins and Hilliard [86] suggested that population sizes should be between 50 and 100 to prevent failures due to bias by the highest fitness individuals. Recent developments in this class of algorithms introduce new techniques to update the covariance matrix of the distribution used to sample new points. Hansen [56] proposed a covariance matrix adaptation method which adapts the resulting search distribution to the contours of the objective function by updating the covariance matrix deterministically using information from evaluated points. The resulting distribution draws new sample points with higher probability in expected promising areas.

\subsubsection{Particle swarm algorithms}

Particle swarm optimization is a population-based algorithm introduced by Kennedy and Eberhart [44,77] that maintains at each iteration a swarm of particles (set of points) with a velocity vector associated with each particle. A new set of particles is produced from the previous swarm using rules that take into account particle swarm parameters (inertia, cognition, and social) and randomly generated weights. Particle swarm optimization has enjoyed recent interest resulting in hybrid algorithms that combine the global scope of the particle swarm search with the faster local convergence of the Nelder-Mead simplex algorithm [46] or GSS methods [138].

\section{Historical overview and some algorithmic insights}

A timeline in the history of innovation in the context of derivative-free algorithms is provided in Fig. 1, while Table 1 lists works that have received over 1,000 citations each. As seen in Fig. 1, early works appeared sparingly between 1960 and 1990. The Hooke-Jeeves and Nelder-Mead algorithms were the dominant approaches in the 1960s and 1970s, and continue to be popular. Stochastic algorithms were introduced in the 1970s and 1980s and have been the most cited. There was relatively little theory behind the deterministic algorithms until the 1990s. Over the last two decades, the emphasis in derivative-free optimization has shifted towards the theoretical understanding of existing algorithms as well as the development of approaches based on surrogate models. The understanding that management of the geometry of surrogate models has a considerable impact on the performance of the underlying algorithms led to the development of several new competing techniques. As seen in Fig. 1, these developments have led to a renewed interest in derivative-free optimization.

\subsection{Algorithmic insights}

The above classification of algorithms to direct and model based, as well as deterministic and stochastic was based on each algorithm's predominant characteristics. Many of the software implementations of these algorithms rely on hybrids that involve characteristics from more than one of the major algorithmic categories. Yet, in all cases, every iteration of a derivative-free method can be viewed as a process the main purpose of which is to determine the next point(s) to evaluate. Information used to make this determination is obtained from a subset of the set of previously evaluated points, ranging from an empty subset to a single previously evaluated point to all previously evaluated points. Different priorities are assigned to potential next points and the selection of the next point(s) to evaluate largely depends on 


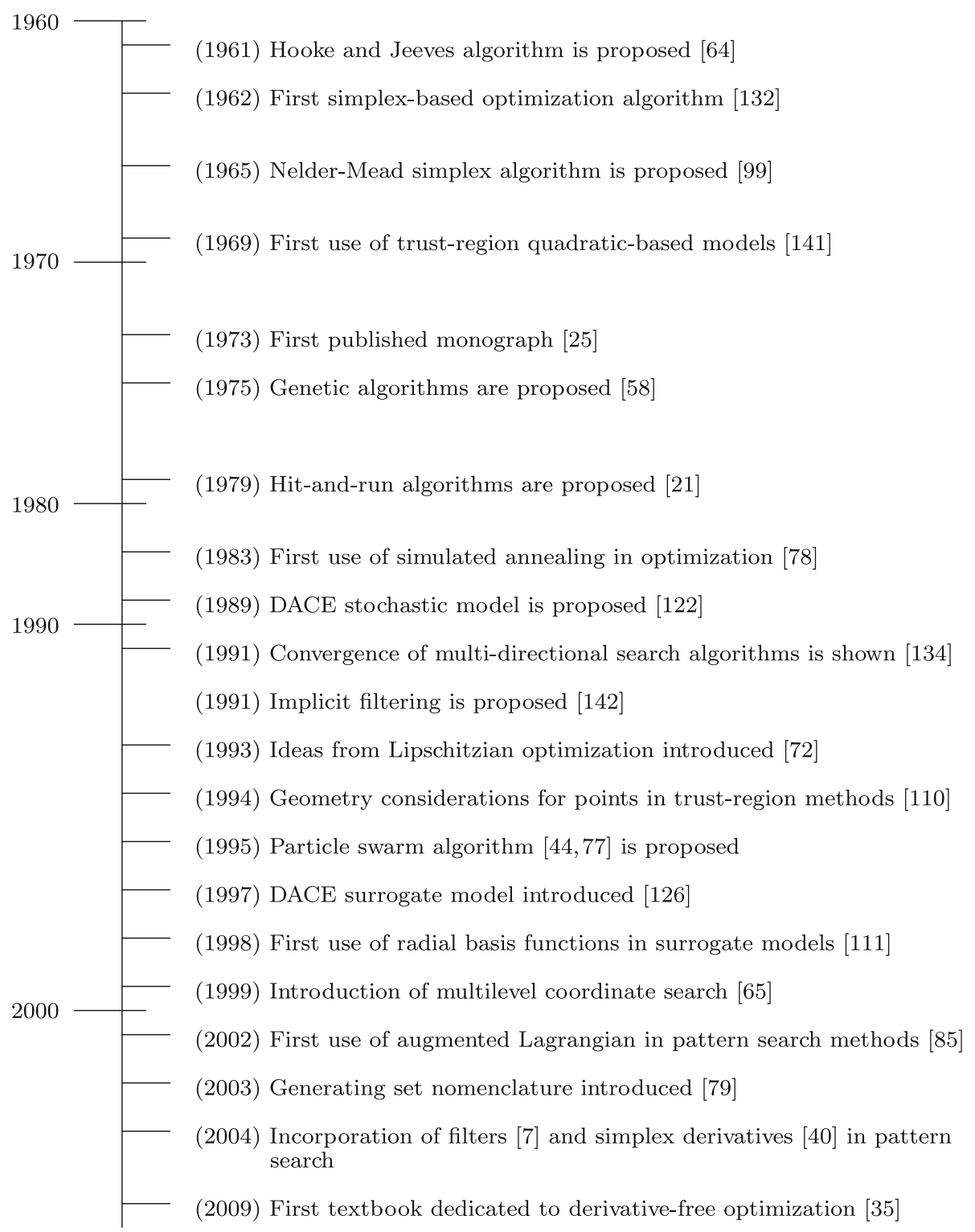

Fig. 1 Timeline of innovation in derivative-free optimization

whether the algorithm partitions the search space into subsets. We thus consider algorithms as belonging to two major groups:

1. Algorithms that do not partition the search space. In this case, the selection step uses information from a subset of points and the next point to be evaluated may be located anywhere in the search space. An example of methods using information from a single point is simulated annealing. In this algorithm, each iteration uses the incumbent to generate a new point through a randomization process. Examples of methods 
Table 1 Citations of most cited works in derivative-free algorithms

a From Google Scholar on 20 December 2011

\begin{tabular}{llr}
\hline Publication & Year appeared & Citations $^{\mathrm{a}}$ \\
\hline Hooke and Jeeves [64] & 1961 & 2,281 \\
Nelder and Mead [99] & 1965 & 13,486 \\
Brent [25] & 1973 & 2,019 \\
Holland [58] & 1975 & 31,494 \\
Kirkpatrick et al. [78] & 1983 & 23,053 \\
Eberhart and Kennedy [44,77] & 1995 & 20,369 \\
\hline
\end{tabular}

using information from multiple previously evaluated points are genetic algorithms, RBF methods, the poll step in pattern search methods, and the Nelder-Mead algorithm. In genetic algorithms, for instance, each iteration considers a set of evaluated points and generates new points through multiple randomization processes. RBF methods generate the next point by optimizing a model of the function created using information from all previously evaluated points. In generating set search methods, each iteration evaluates points around the current iterate in directions generated and ordered using information from previously evaluated points. These directions are usually required to be positive spanning sets in order to guarantee convergence via a sequence of positive steps over these directions. MADS methods use information from previously evaluated points to produce the best search directions out of an infinite set of search directions. Finally, the Nelder-Mead algorithm generates the next point by considering information of the geometry and objective values of a set of previously evaluated points.

2. Algorithms that partition the search space. In this case, partition elements are assigned priorities used later to select the most promising partition elements. The methods that rely on partitioning include direct methods, such as DIRECT, the model-based trust-region algorithms, the largely direct approach of MCS, which also incorporates model-based local search, and approaches akin to branch-and-bound. In DIRECT, the partitions are generated in a way that evaluated points lie at the center of partition elements. MCS, on the other hand allows evaluated points on the boundary of partition elements. While DIRECT does not use an explicit model of $f$ and MCS involves local quadratic models of $f$, branch-and-bound combines partitioning with models of the gradient of $f$. Finally, trust-region methods use information from multiple previously evaluated points. These methods partition the space to two subsets that change over the course of the algorithm: a region of interest (the trust region) in the neighborhood of the current iterate and its complement. The next point to be evaluated is typically obtained by optimizing an interpolating model using information from a subset of previously evaluated points.

\section{Derivative-free optimization software}

The purpose of this and the following two sections is to examine the status of software implementations of the algorithms reviewed above. The software implementations for which results are presented here have been under development and/or released since 1998. They are all capable of dealing with black-box functions in a non-intrusive way, i.e., the source code of the optimization problem to be solved is assumed to be unavailable or impractical to modify. Table 2 lists the solvers considered, along with their main characteristics. Each 
Table 2 Derivative-free solvers considered in this paper

\begin{tabular}{|c|c|c|c|c|c|}
\hline Solver & URL & Version & Language & Bounds & Constraints \\
\hline ASA & www.ingber.com & 26.30 & $\mathrm{C}$ & Required & No \\
\hline BOBYQA & $\begin{array}{l}\text { Available by email from } \\
\text { mjdp@cam.ac.uk }\end{array}$ & 2009 & Fortran & Required & No \\
\hline CMA-ES & $\begin{array}{l}\text { www.lri.fr/ hansen/cmaesintro. } \\
\text { html }\end{array}$ & 3.26beta & Matlab & Optional & No \\
\hline DAKOTA/DIRECT & www.cs.sandia.gov/dakota/ & 4.2 & $\mathrm{C}++$ & Required & Yes \\
\hline DAKOTA/EA & www.cs.sandia.gov/dakota/ & 4.2 & $\mathrm{C}++$ & Required & Yes \\
\hline DAKOTA/PATTERN & www.cs.sandia.gov/dakota/ & 4.2 & $\mathrm{C}++$ & Required & Yes \\
\hline DAKOTA/SOLIS-WETS & www.cs.sandia.gov/dakota/ & 4.2 & $\mathrm{C}++$ & Required & Yes \\
\hline $\mathrm{DFO}$ & projects.coin-or.org/Dfo & 2.0 & Fortran & Required & Yes \\
\hline FMINSEARCH & www.mathworks.com & 1.1.6.2 & Matlab & No & No \\
\hline GLOBAL & www.inf.u-szeged.hu/ csendes & 1.0 & Matlab & Required & No \\
\hline HOPSPACK & software.sandia.gov/trac/hopspack & 2.0 & $\mathrm{C}++$ & Optional & Yes \\
\hline IMFIL & www4.ncsu.edu/ ctk/imfil.html & 1.01 & Matlab & Required & Yes \\
\hline MCS & $\begin{array}{l}\text { www.mat.univie.ac.at/ neum/ } \\
\text { software/mcs/ }\end{array}$ & 2.0 & Matlab & Required & No \\
\hline NEWUOA & $\begin{array}{l}\text { Available by email from } \\
\text { mjdp@cam.ac.uk }\end{array}$ & 2004 & Fortran & No & No \\
\hline NOMAD & www.gerad.ca/nomad/ & 3.3 & $\mathrm{C}++$ & Optional & Yes \\
\hline PSWARM & www.norg.uminho.pt/aivaz/pswarm/ & 1.3 & Matlab & Required & Yes $^{\mathrm{a}}$ \\
\hline SID-PSM & www.mat.uc.pt/sid-psm/ & 1.1 & Matlab & Optional & Yes $^{\mathrm{a}}$ \\
\hline SNOBFIT & $\begin{array}{l}\text { www.mat.univie.ac.at/ neum/ } \\
\text { software/snobfit/ }\end{array}$ & 2.1 & Matlab & Required & No \\
\hline TOMLAB / GLCCLUSTER & tomopt.com & 7.3 & Matlab & Required & Yes \\
\hline TOMLAB / LGO & www.pinterconsulting.com/ & 7.3 & Matlab & Required & Yes \\
\hline TOMLAB / MULTIMIN & tomopt.com & 7.3 & Matlab & Required & Yes \\
\hline TOMLAB / OQNLP & tomopt.com & 7.3 & Matlab & Required & Yes \\
\hline
\end{tabular}

a Handles linear constraints only

solver is discussed in detail in the sequel. The column 'Bounds' refers to the ability of solvers to handle bounds on variables. Possible entries for this column are "no" for solvers that do not allow bounds; "required" for solvers that require bounds on all variables; and "optional" otherwise. The column 'Constraints' refers to the ability of solvers to handle linear algebraic constraints and general constraints that are available as a black-box executable but not in functional form.

\subsection{ASA}

Adaptive Simulated Annealing (ASA) [68] is a C implementation developed for unconstrained optimization problems. ASA departs from traditional simulated annealing in that it involves a generating probability density function with fatter tails than the typical Boltzmann distribution. This allows ASA to possibly escape from local minima by considering points far away from the current iterate. Separate temperature parameters are assigned for each variable and they are updated as the optimization progresses. 


\subsection{BOBYQA}

Bound Optimization BY Quadratic Approximation (BOBYQA) is a Fortran implementation of Powell's model-based algorithm [115]. BOBYQA is an extension of the NEWUOA algorithm to bounded problems with additional considerations on the set of rules that maintain the set of interpolating points.

\subsection{CMA-ES}

Covariance Matrix Adaptation Evolution Strategy (CMA-ES) [55] is a genetic algorithm implemented in multiple languages including C, Matlab, and Python. Mutation is performed by a perturbation with expected value zero and a covariance matrix which is iteratively updated to guide the search towards areas with expected lower objective values [56].

\subsection{DAKOTA solvers}

Design Analysis Kit for Optimization and Terascale Applications (DAKOTA) [45] is a project at Sandia National Laboratories. DAKOTA's initial scope was to create a toolkit of blackbox optimization methods. The scope was later expanded to include additional optimization methods and other engineering applications, including design of experiments, and nonlinear least squares.

DAKOTA contains a collection of optimization software packages featuring the COLINY library [124] that includes, among others, the following solvers that we tested:

1. DAKOTA/EA: an implementation of various genetic algorithms;

2. DAKOTA/DIRECT: an implementation of the DIRECT algorithm;

3. DAKOTA/PATTERN: various pattern search methods; and

4. DAKOTA/SOLIS-WETS: greedy search, comparing the incumbent with points generated from a multivariate normal distribution.

\subsection{DFO}

Derivative Free Optimization (DFO) $[28,125]$ is an open-source Fortran implementation of the trust-region-based algorithm originally developed by Conn et al. [31,32] and expanded by Conn et al. [33]. DFO is a local optimization method designed to handle very expensive function evaluations for small-dimensional problems with fewer than 50 variables. Given a set of points, DFO identifies the point with the best objective found and builds a quadratic model by interpolating a selected subset of points. The resulting model is optimized within a trust region centered at the best point. Our computational experiments used the open-source Fortran software IPOPT [29] to solve the trust-region subproblems.

\subsection{FMINSEARCH}

FMINSEARCH is an implementation of the Nelder-Mead simplex-based method of Lagarias et al. [81]. This code is included as a Matlab built-in function in the Optimization Toolbox and handles unconstrained optimization problems. 


\subsection{GLOBAL}

GLOBAL [37] is a Matlab implementation of a multistart stochastic method proposed by Boender et al. [20]. GLOBAL draws random uniformly distributed points in a space of interest, selects a sample, and applies a clustering procedure. The derivative-free local search solver UNIRANDI [69], based on random direction generation and linear search, is used from points outside the cluster.

\subsection{HOPSPACK}

Hybrid Optimization Parallel Search PACKage (HOPSPACK) [108] is a parallel processing framework implemented in $\mathrm{C}++$ that includes a GSS local solver. The user is allowed to perform an asynchronous parallel optimization run using simultaneously the embedded GSS local solver, along with user-provided solvers.

\subsection{IMFIL}

IMFIL [74] is a Matlab implementation of the implicit filtering algorithm [50,142].

\subsection{MCS}

Multilevel coordinate search (MCS) [101] is a Matlab implementation of the algorithm proposed by Huyer and Neumaier [65] for global optimization of bound-constrained problems.

\subsection{NEWUOA}

NEWUOA [114] is a Fortran implementation of Powell's model-based algorithm for derivative-free optimization [113]. The inputs to NEWUOA include initial and lower bounds for the trust-region radius, and the number of function values to be used for interpolating the quadratic model.

\subsection{NOMAD}

NOMAD [6,82] is a $\mathrm{C}++$ implementation of the LTMADS [11] and ORTHO-MADS [8] methods with the extreme barrier [11], filter [2] and progressive barrier [12] approaches to handle general constraints. It is designed to solve nonlinear, nonsmooth, noisy optimization problems. NOMAD's termination criterion allows for a combination of the number of points evaluated, minimum mesh size, and the acceptable number of consecutive trials that fail to improve the incumbent.

A related implementation, NOMADm [3], is a collection of Matlab functions that solve bound-constrained, linear or nonlinear optimization problems with continuous, discrete, and categorical variables.

\subsection{PSWARM}

PSWARM [137] is a C implementation of the particle swarm pattern search method [138]. Its search step performs global search based on the particle swarm algorithm. Its poll step relies on a coordinate search method. The poll directions coincide with positive and negative unit vectors of all variable axes. 
PSWARM allows the user to compile and use the solver as a stand-alone software or as a custom AMPL solver. A related Matlab implementation PSwarmM is also available [137].

\subsection{SID-PSM}

SID-PSM [41] is a Matlab implementation of a pattern search method with the poll step guided by simplex derivatives [40]. The search step relies on the optimization of quadratic surrogate models [39]. SID-PSM is designed to solve unconstrained and constrained problems.

\subsection{SNOBFIT}

SNOBFIT is a Matlab implementation of the branch-and-fit algorithm proposed by Huyer and Neumaier [66].

\subsection{TOMLAB solvers}

TOMLAB [60] is a Mat lab environment that provides access to several derivative-free optimization solvers, the following of which were tested:

1. TOMLAB/GLCCLUSTER [60, pp. 109-111]: an implementation of the DIRECT algorithm [71] hybridized with clustering techniques [59];

2. TOMLAB / LGO [107]: a suite of global and local nonlinear solvers [106] that implements a combination of Lipschitzian-based branch-and-bound with deterministic and stochastic local search (several versions of LGO are available, for instance under Maple, and may offer different features than TOMLAB / LGO);

3. TOMLAB /MULTIMIN [60, pp. 148-151]: a multistart algorithm; and

4. TOMLAB / OQNLP [62]: a multistart approach that performs searches using a local NLP solver from starting points chosen by a scatter search algorithm.

\subsection{Additional solvers considered}

In addition to the above solvers for which detailed results are presented in the sequel, we experimented with several solvers for which results are not presented here. These solvers were:

1. PRAXIS: an implementation of the minimization algorithm of Brent [25];

2. TOMLAB/GLBSOLVE [60, pp. 106-108]: an implementation of the DIRECT algorithm [72], specifically designed to handle box-bounded problems;

3. TOMLAB / GLCSOLVE [60, pp. 118-122]: an implementation of an extended version of the DIRECT algorithm [72] that can handle integer variables and linear or nonlinear constraints;

4. TOMLAB / EGO [61, pp. 11-24]: an implementation of the EGO algorithm [73,126] modified to handle linear and nonlinear constraints;

5. TOMLAB/RBFSOLVE [61, pp. 5-10]: an implementation of the radial basis function $[19,53]$ that can handle box-constrained global optimization problems.

The PRAXIS solver is one of the first derivative-free optimization solvers developed. This solver had below average performance and has not been updated since its release in 1973. Results for the above four TOMLAB solvers are not included in the comparisons since these solvers have been designed with the expectation for the user to provide a reasonably small bounding box for the problem variables [59]. 


\section{Illustrative example: camel6}

To illustrate and contrast the search strategies that are employed by different algorithms, Fig. 2 shows how the different solvers progress for came16. This two-dimensional test problem, referred to as the 'six-hump camel back function,' exhibits six local minima, two of which are global minima. In the graphs of Fig. 2, red and blue are used to represent high and low objective function values, respectively. Global minima are located at $[-0.0898,0.7126]$
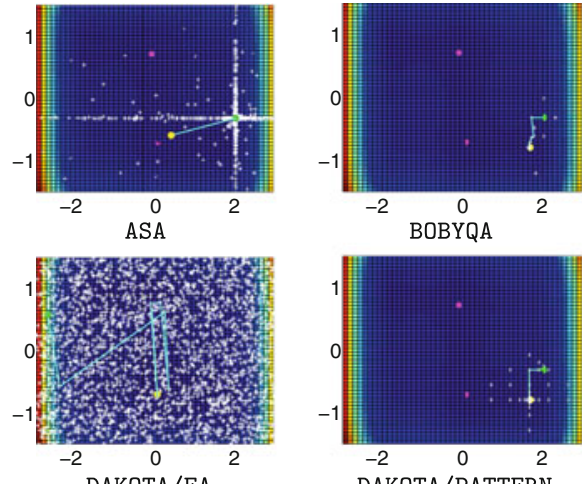

DAKOTA/EA

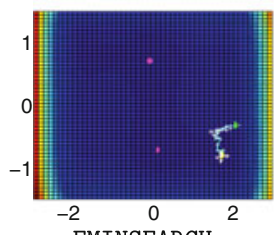

FMINSEARCH
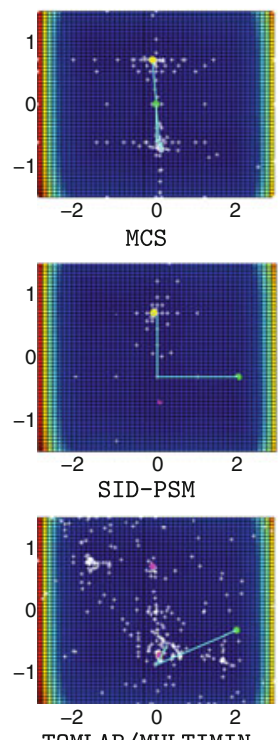

TOMLAB/MULTIMIN
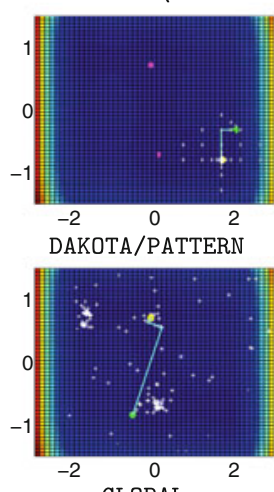

GLOBAL
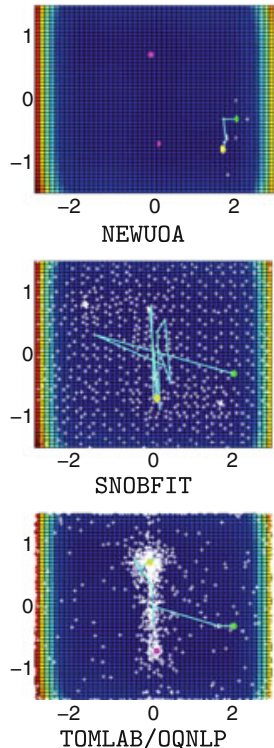

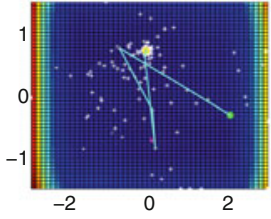

CMA-ES

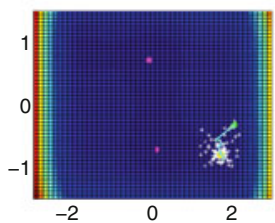

DAKOTA/SOLIS-WETS

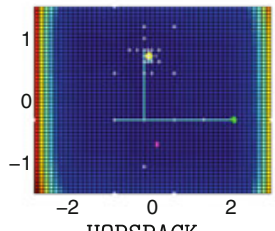

HOPSPACK
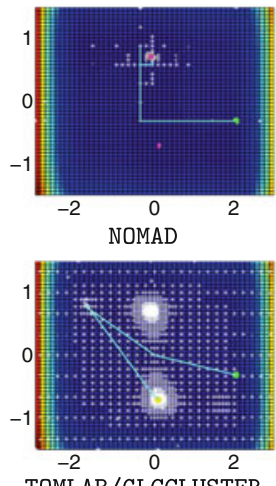

TOMLAB/GLCCLUSTER

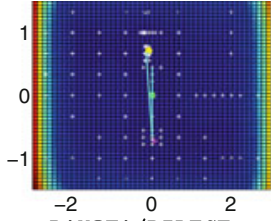

DAKOTA/DIRECT
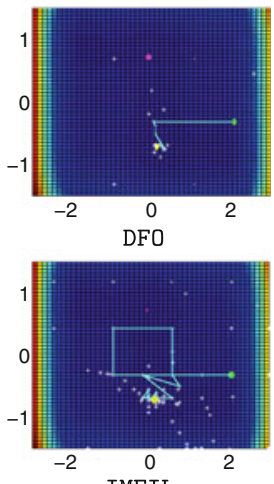

IMFIL
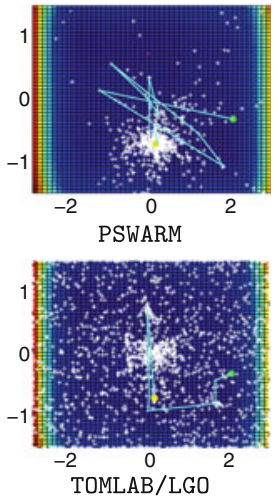

Fig. 2 Solver search progress for test problem came16 
and $[0.0898,-0.7126]$ and are marked with magenta circles. Each solver was given a limit of 2,500 function evaluations and the points evaluated are marked with white crosses. Solvers that require a starting point were given the same starting point. Starting points are marked with a green circle. The trajectory of the progress of the best point is marked with a cyan line, and the final solution is marked with a yellow circle. As illustrated by these plots, solvers DAKOTA/ PATTERN, DAKOTA/SOLIS-WETS, FMINSEARCH, and NEWUOA perform a local search, exploring the neighborhood of the starting point and converging to a local minimum far from the global minima. DIRECT-based methods DAKOTA/DIRECT and TOMLAB / GLCCLUSTER perform searches that concentrate evaluated points around the local minima. Indeed, the two global minima are found by these solvers.

It is clear from Fig. 2 that the stochastic solvers CMA-ES, DAKOTA/EA, and PSWARM perform a rather large number of function evaluations that cover the entire search space, while local search algorithms terminate quickly after improving the solution of the starting point locally. Partitioning-based solvers seem to strike a balance by evaluating more points than local search algorithms but fewer than stochastic search approaches.

\section{Computational comparisons}

\subsection{Test problems}

As most of the optimization packages tested in this study were designed for low-dimensional unconstrained problems, the problems considered were restricted to a maximum of 300 variables with bound constraints only. The solvers were tested on the following problems:

1. Richtarik's [119] piece-wise linear problems:

$$
\min _{x} \max _{i}\left\{\left|\left\langle a_{i}, x\right\rangle\right|: i=1,2, \ldots, m\right\},
$$

2. Nesterov's [100] quadratic test problems:

$$
\min _{x} \frac{1}{2}\|A x-b\|_{2}^{2}+\|x\|_{1},
$$

3. a variant of Nesterov's test problems without the nonsmooth term:

$$
\min _{x} \frac{1}{2}\|A x-b\|_{2}^{2}
$$

4. the ARWHEAD test problem from Conn et al. [30]:

$$
\min _{x} \sum_{i=1}^{n-1}\left(x_{i}^{2}+x_{n}^{2}\right)^{2}-4 x_{i}+3,
$$

5. 245 nonconvex problems from the globallib [51] and princetonlib [116],

6. and 49 nonsmooth problems from the collection of Lukšan and Vlček [89].

For the first four families of problems, instances were generated with sizes of 5, 10, 15, $20,25,30,35,40,45,50,100,200$, and 300 variables. For each problem size, five random instances were generated for Richtarik's and both variants of Nesterov's problems.

We use the number of variables to classify each problem in one of four groups as shown in Table 3. The test problems of Table 3 are diverse, involving sums of squares problems, quadratic and higher degree polynomials, continuous and discontinuous functions, 32 problems 
Table 3 Characteristics of test problems

\begin{tabular}{|c|c|c|c|c|c|c|c|c|}
\hline \multirow[t]{2}{*}{$n$} & \multicolumn{3}{|c|}{ Number of convex problems } & \multicolumn{3}{|c|}{ Number of nonconvex problems } & \multirow[t]{2}{*}{ Total } & \multirow[t]{2}{*}{$n_{\text {avg }}$} \\
\hline & Smooth & Non-smooth & Total & Smooth & Non-smooth & Total & & \\
\hline $1-2$ & 0 & 9 & 9 & 86 & 4 & 90 & 99 & 1.9 \\
\hline $3-9$ & 6 & 19 & 25 & 97 & 11 & 108 & 133 & 5.1 \\
\hline $10-30$ & 30 & 59 & 89 & 27 & 3 & 30 & 119 & 18.5 \\
\hline $31-300$ & 42 & 74 & 116 & 35 & 0 & 35 & 153 & 104.6 \\
\hline $1-300$ & 78 & 161 & 239 & 245 & 18 & 263 & 502 & 37.6 \\
\hline
\end{tabular}

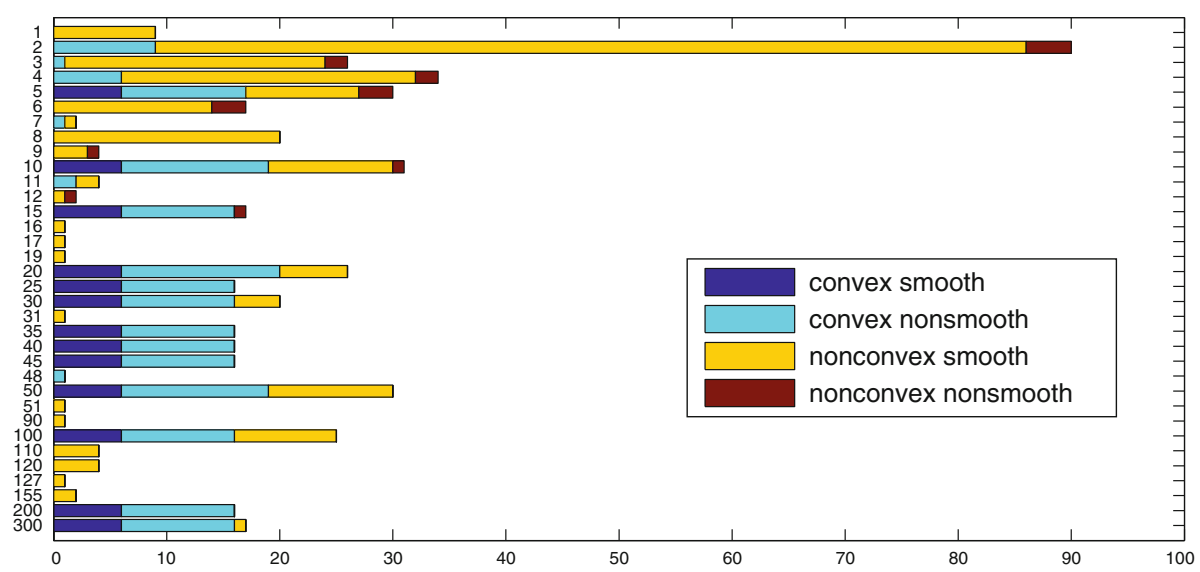

Fig. 3 Number of variables versus number of test problems

with trigonometric functions, and 33 problems with exponential or logarithmic functions. A total of 239 of the test problems are convex, while 263 are non-convex. The number of variables $(n)$ ranged from 1 to 300 , with an average number of variables $\left(n_{\text {avg }}\right)$ equal to 37.6. Figure 3 presents the distribution of problems by dimensionality and by problem class.

All test problems are available at http://archimedes.cheme.cmu.edu/?q=dfocomp. The same web site provides detailed results from the application of the different solvers to the test problems.

\subsection{Experimental setup and basis of solver comparisons}

All computations were performed on Intel $2.13 \mathrm{GHz}$ processors running Linux and Mat lab R2 010a. The 22 solvers of Table 2 were tested using a limit of 2,500 function evaluations in each run. To put this limit in perspective, 2,500 function evaluations for the bioremedation model of [98], which represents a typical application of derivative-free optimization methods to expensive engineering problems, would run for about 200 CPU days.

Variable bounds are required by many of the solvers but were not available for many test problems. For problems that lacked such bounds in the problem formulation, we restricted all variables to the interval $[-10,000,10,000]$ unless these bounds resulted in numerical difficulties due to overflowing. In such cases, tighter bounds were used, provided that they still included the best known solution for each problem. The same variable bounds were 
Table 4 Bounds on test problems

\begin{tabular}{|c|c|c|c|c|c|c|c|}
\hline \multirow{3}{*}{$\begin{array}{l}\text { Available } \\
\text { bounds }\end{array}$} & \multicolumn{6}{|c|}{ Number of problems } & \multirow[t]{3}{*}{ Tota } \\
\hline & \multicolumn{3}{|l|}{ Convex } & \multicolumn{3}{|c|}{ Nonconvex } & \\
\hline & Smooth & Nonsmooth & Total & Smooth & Nonsmooth & Total & \\
\hline Both & 0 & 5 & 5 & 52 & 3 & 55 & 60 \\
\hline Lower & 0 & 72 & 72 & 9 & 4 & 13 & 85 \\
\hline None & 78 & 84 & 162 & 184 & 11 & 195 & 357 \\
\hline Total & 78 & 161 & 239 & 245 & 18 & 263 & 502 \\
\hline
\end{tabular}

used for all solvers. For solvers that do not accept bounds, a large objective function value was returned for argument values outside the bounding box. This value was constant for all iterations and solvers. Whenever starting points were required, they were drawn from a uniform distribution from the box-bounded region. The same randomly generated starting points were used for all solvers. Table 4 presents the number of 'bounded' test problems that came with lower and upper bounds for all variables, the number of test problems that came with lower bounds only, and 'unbounded' test problems that lacked a lower and upper bounds for at least one variable.

Only objective function values were provided to all solvers. The only exception was SID-PSM, which requires the gradient of the constraints. As the problems considered here were bound-constrained with no additional constraints, the gradients provided to SID-PSM were simply a set of unit vectors.

Many of the test problems are nonconvex and most of the solvers tested are local solvers. Even for convex problems, performance of a solver is often affected by the starting point chosen. For this reason, solvers that permitted the use of a starting point were run once from each of ten different starting points. This was possible for all solvers with the exception of DAKOTA/DIRECT, DAKOTA/EA, GLOBAL, and MCS. The latter solvers override the selection of a starting point and start from the center of the box-bounded region. This resulted in a total number of 112,448 optimization instances to be solved.

In order to assess the quality of the solutions obtained by different solvers, we compared the solutions returned by the solvers against the globally optimal solution for each problem. A solver was considered to have successfully solved a problem during a run if it returned a solution with an objective function value within $1 \%$ or 0.01 of the global optimum, whichever was larger. In other words, a solver was considered successful if it reported a solution $y$ such that $f(y) \leq \max \left(1.01 f\left(x^{*}\right), f\left(x^{*}\right)+0.01\right.$, where $x^{*}$ is a globally optimal solution for the problem. To obtain global optimal solutions for the test problems, we used the generalpurpose global optimization solver BARON $[123,133]$ to solve as many of the test problems as possible. Unlike derivative-free solvers, BARON requires explicit algebraic expressions rather than function values alone. BARON's branch-and-bound strategy was able to guarantee global optimality for most of the test problems, although this solver does not accept trigonometric and some other nonlinear functions. For the latter problems, LINDOGLOBAL [87] was used to obtain a global solution.

In comparing the quality of solutions returned, we will compare the average- as well as best-case behavior of each solver. For the average-case behavior, we compare solvers using for each solver the median objective function value of the ten different runs. For the best-case comparison, we compare the best solution found by each solver after all ten runs. 


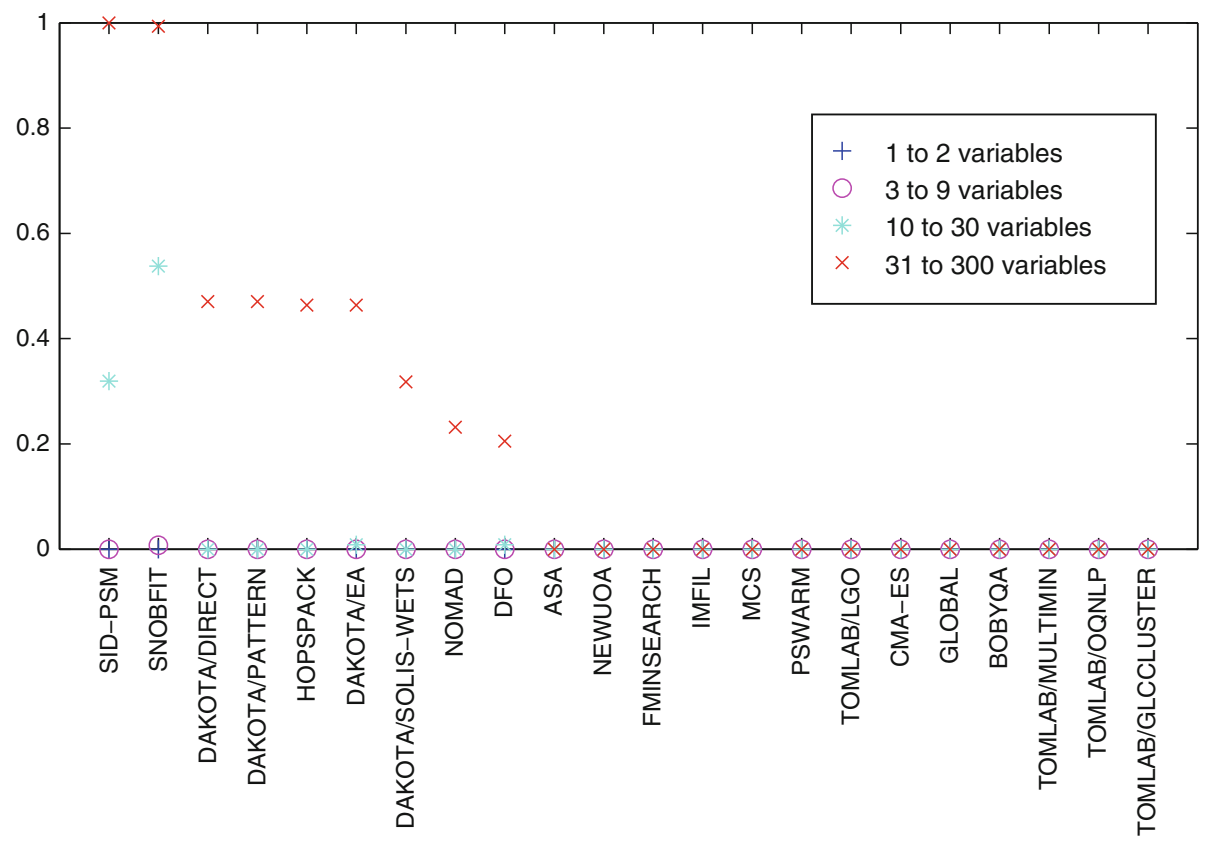

Fig. 4 Fraction of problems over the $10 \mathrm{CPU}$ minute limit

Average-case behavior is presented in the figures and analyzed below unless explicitly stated otherwise.

Most instances were solved within a few minutes. Since the test problems are algebraically and computationally simple and small, the total time required for function evaluations for all runs was negligible. Most of the CPU time was spent by the solvers on processing function values and determining the sequence of iterates. A limit of $10 \mathrm{CPU}$ minutes was imposed on each run. Figure 4 presents the fraction of problems of different size that were terminated at any of the 10 optimization instances after reaching the CPU time limit. As seen in this figure, no solver reached this CPU time limit for problems with up to nine variables. For problems with ten to thirty variables, only SID-PSM and SNOBFIT had to be terminated because of the time limit. These two solvers also hit the time limit for all problems with more than thirty variables, along with seven additional solvers.

\subsection{Algorithmic settings}

Algorithmic parameters for the codes under study should be chosen in a way that is reflective of the relative performance of the software under consideration. Unfortunately, the optimization packages tested have vastly different input parameters that may have a significant impact upon the performance of the algorithm. This presents a major challenge as a computational comparison will have to rely on a few choices of algorithmic parameters for each code. However, for expensive experiments and time-demanding simulations like the bioremedation model of [98], practitioners cannot afford to experiment with many different algorithmic options. Even for less expensive functions, most typical users of optimization packages are not experts on the theory and implementation of the underlying algorithm and rarely explore software options. Thus, following the approach of [102] in a recent compari- 


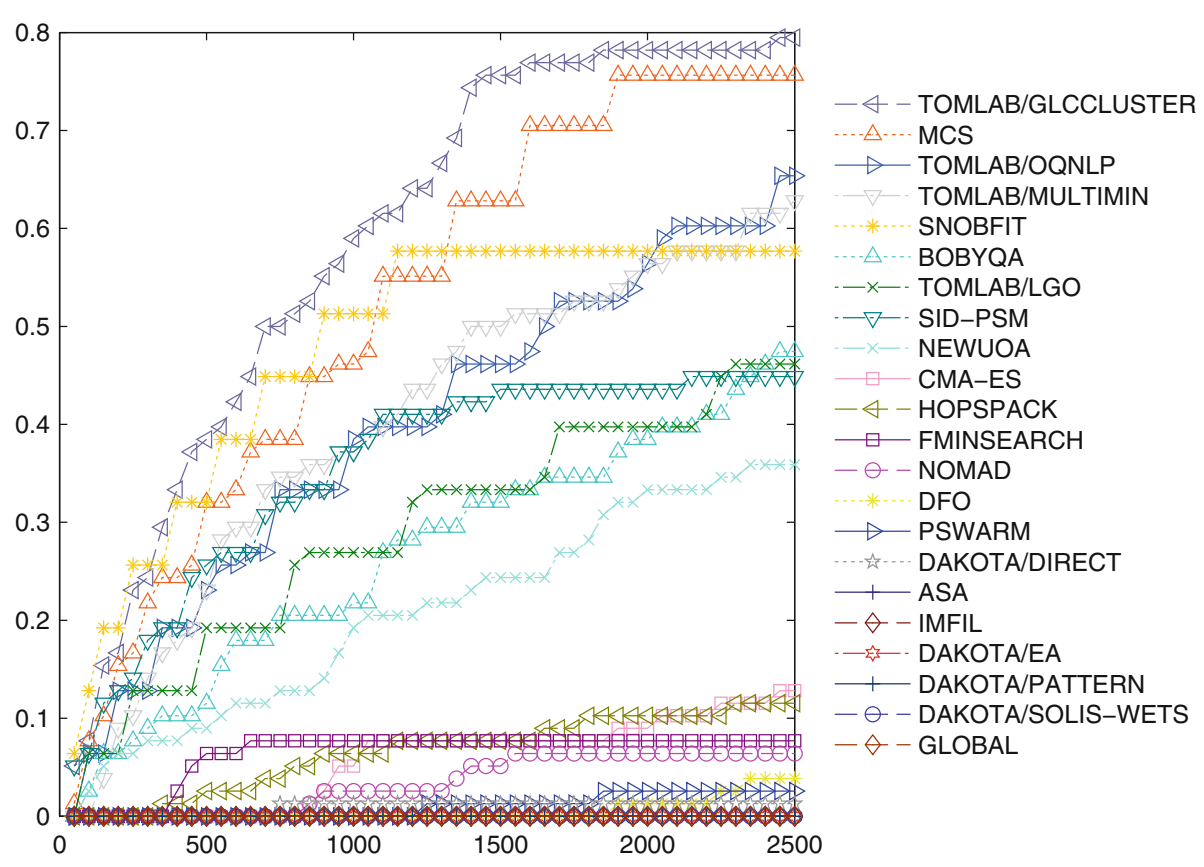

Fig. 5 Fraction of convex smooth problems solved as a function of allowable number of function evaluation

son of optimization codes, comparisons were carried out using the default parameter values for each package, along with identical stopping criteria and starting points across solvers. Nonetheless, all software developers were provided with early results of our experiments and given an opportunity to revise or specify their default option values.

Optimization instances in which a solver used fewer function evaluations than the imposed limit were not pursued further with that particular solver. In practice, a user could employ the remaining evaluations to restart the solver but this procedure is highly user-dependent. Our experiments did not use restart procedures in cases solvers terminated early.

\subsection{Computational results for convex problems}

Figure 5 presents the fraction of convex smooth problems solved by each solver to within the optimality tolerance. The horizontal axis shows the progress of the algorithm as the number of function evaluations gradually reached 2,500. The best solver, TOMLAB / GLCCLUSTER, solved $79 \%$ of convex smooth problems, closely followed by MCS which solved $76 \%$, and SNOBF IT, TOMLAB / OQNLP, and TOMLAB / MULTIMIN all of which solved over $58 \%$ of the problems. The solvers ASA, DAKOTA/EA, DAKOTA/PATTERN, DAKOTA/SOLIS-WETS, GLOBAL, and IMFIL did not solve any problems within the optimality tolerance. Figure 6 presents the fraction of convex nonsmooth problems solved. At 44 and $43 \%$ of the convex nonsmooth problems solved, TOMLAB/MULTIMIN and TOMLAB/GLCCLUSTER have a significant lead over all other solvers. TOMLAB / LGO and TOMLAB / OQNLP follow with 22 and $20 \%$, respectively. Fifteen solvers are not even able to solve $10 \%$ of the problems. It is strange that model-based solvers, which have nearly complete information for many of the 


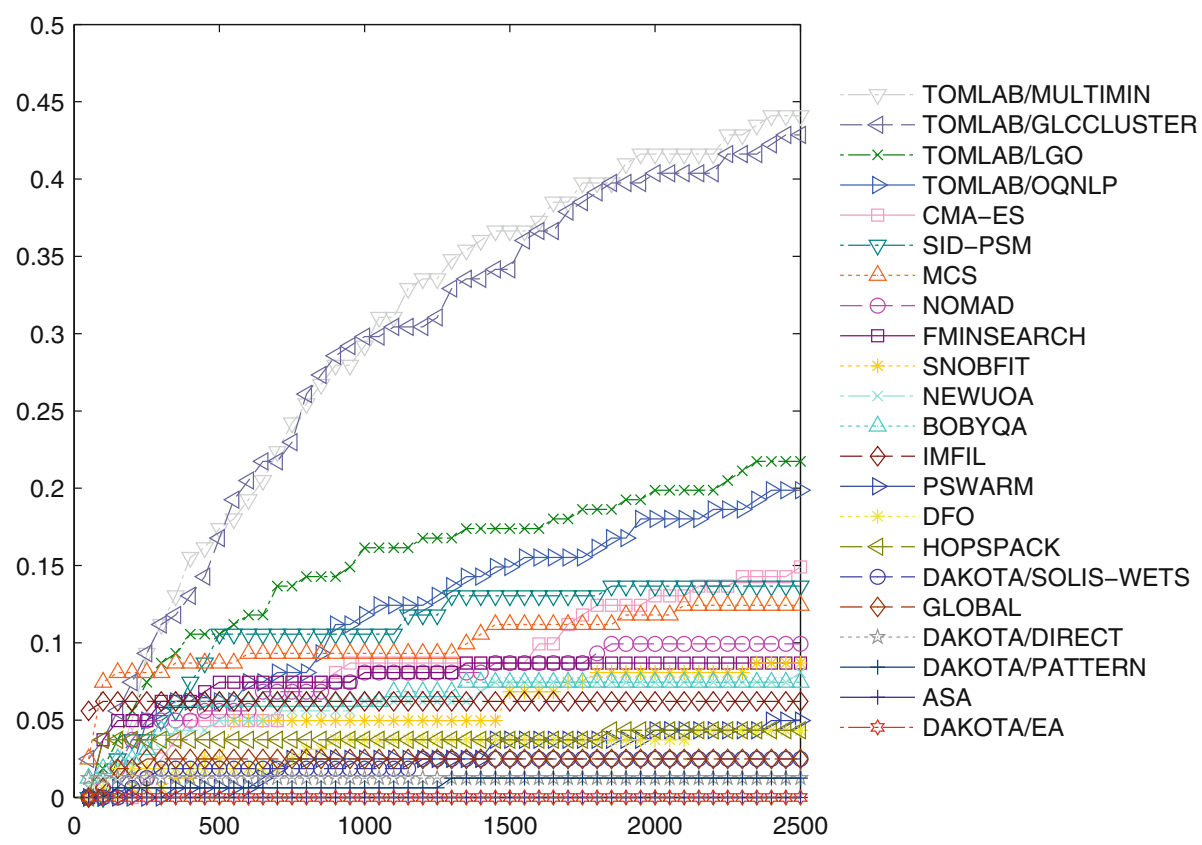

Fig. 6 Fraction of convex nonsmooth problems solved as a function of allowable number of function evaluations

tested problems, solve a small fraction of problems. However, some of these solvers are old and most of them are not extensively tested.

A somewhat different point of view is taken in Figs. 7 and 8, where we present the fraction of problems for which each solver achieved a solution as good as the best solution among all solvers, without regard to the best known solution for the problems. When multiple solvers achieved the same solution, all of them were credited as having the best solution among all solvers. As before, the horizontal axis denotes the number of allowable function evaluations.

Figure 7 shows that, for convex smooth problems, TOMLAB / MULTIMIN has a brief lead until 200 function calls, at which point TOMLAB/GLCCLUSTER takes the lead, finishing with $81 \%$. TOMLAB / MULTIMIN and MCS follow closely at around $76 \%$. The performance of TOMLAB/MULTIMIN, MCS and TOMLAB/OQNLP improves with the number of allowable function evaluations. Ten solvers are below the $10 \%$ mark, while five solvers did not find a best solution for any problem for any number of function calls.

Similarly, Fig. 8 shows that, for convex nonsmooth problems, the solver TOMLAB / MULTIMIN leads over the entire range of function calls, ending at 2,500 function evaluations with the best solution for $66 \%$ of the problems. TOMLAB / GLCCLUSTER follows with the best solution for $52 \%$ of the problems. There is a steep difference with the remaining twenty solvers, which, with the exception of TOMLAB / LGO, TOMLAB / OQNLP, CMA-ES and SID-PSM, are below the $10 \%$ mark.

An interesting conclusion from Figs. 5, 6, 7, and 8 is that, with the exception of NEWUOA and BOBYQA for convex smooth problems, local solvers do not perform as well as global solvers do even for convex problems. By casting a wider net, global solvers are able to find better solutions than local solvers within the limit of 2,500 function calls. 


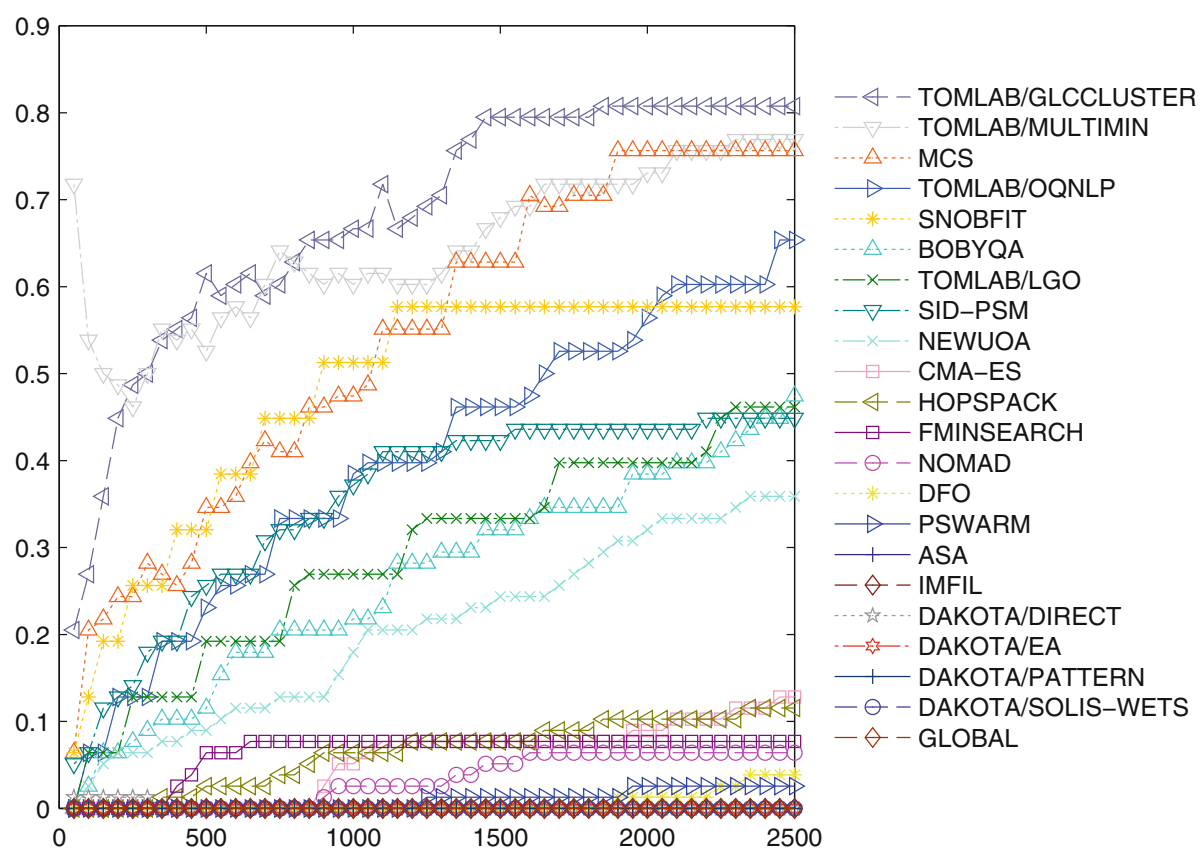

Fig. 7 Fraction of convex smooth problems, as a function of allowable number of function evaluations, for which a solver found the best solution among all solvers

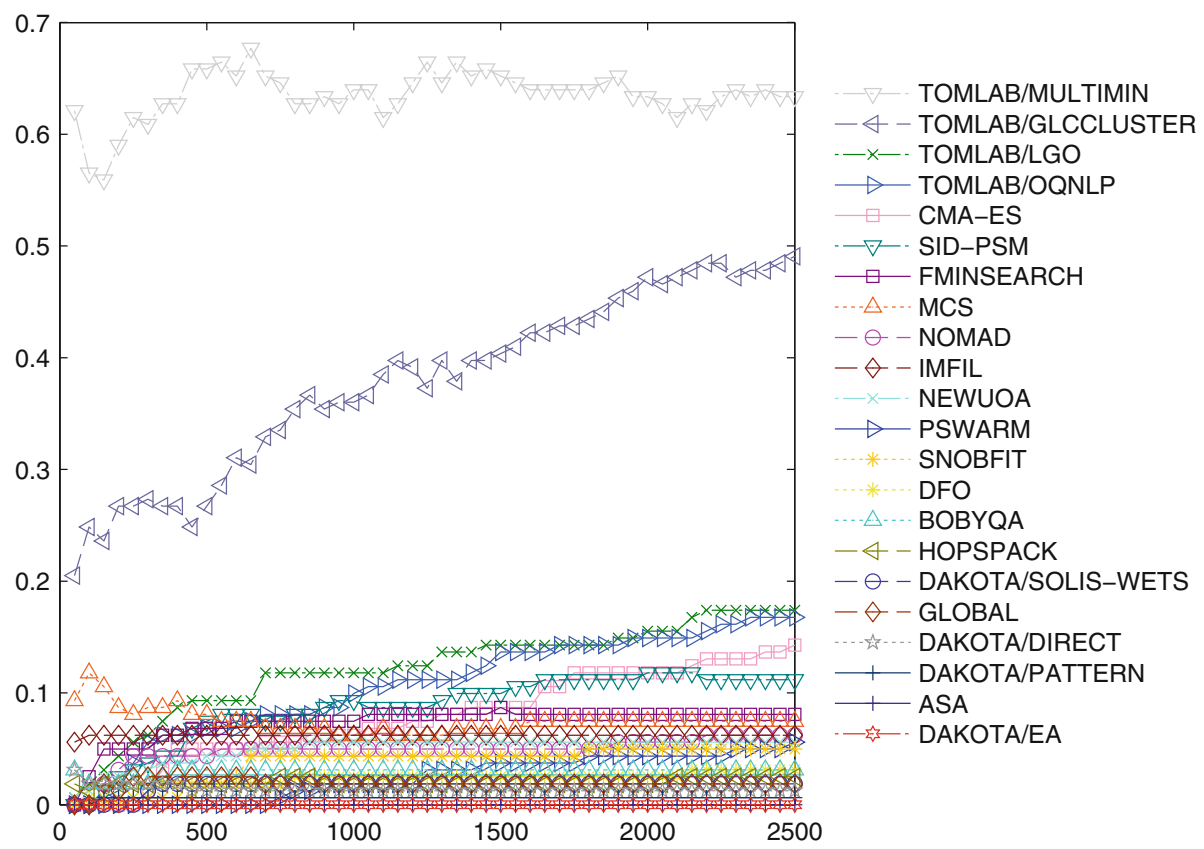

Fig. 8 Fraction of convex nonsmooth problems, as a function of allowable number of function evaluations, for which a solver found the best solution among all solvers 


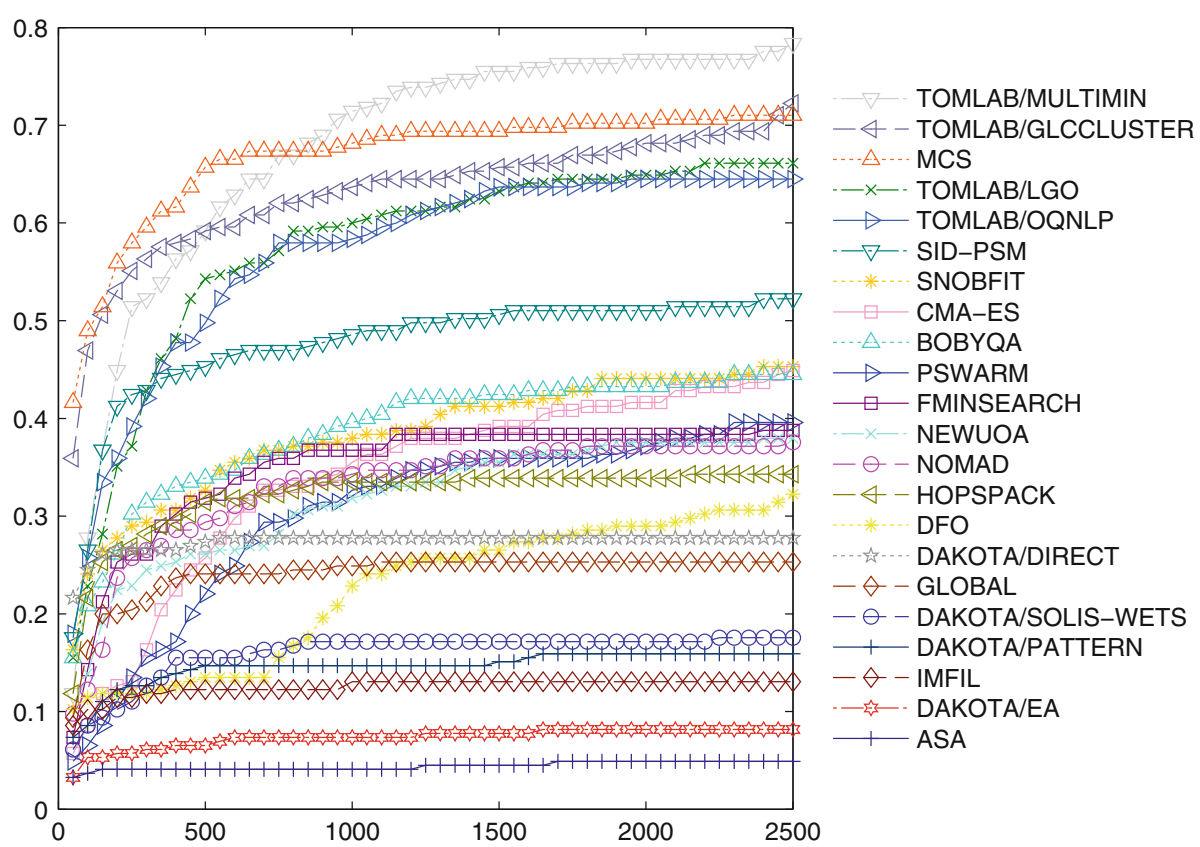

Fig. 9 Fraction of nonconvex smooth problems solved as a function of allowable number of function evaluations

\subsection{Computational results with nonconvex problems}

Figure 9 presents the fraction of nonconvex test problems for which the solver median solution was within the optimality tolerance from the best known solution. As shown in this figure, MCS (up to 800 function evaluations and TOMLAB/MULTIMIN (beyond 800 function evaluations) attained the highest percentage of global solutions, solving over $70 \%$ of the problems at 2,500 function evaluations. The group of top solvers also includes TOMLAB/GLCCLUSTER, TOMLAB/LGO and TOMLAB/OQNLP, which found over $64 \%$ of the global solutions. Nine solvers solved over $44 \%$ of the cases, and only two solvers could not find the solution for more than $10 \%$ of the cases. CMA-ES returned the best results among the stochastic solvers.

At a first glance, it may appear surprising that the percentage of nonconvex smooth problems solved by certain solvers (Fig. 9) exceeds the percentage of convex ones (Fig. 5). Careful examination of Table 3, however, reveals that the nonconvex problems in the test set contain, on average, fewer variables.

Figure 10 presents the fraction of nonconvex nonsmooth test problems for which the solver median solution was within the optimality tolerance from the best known solution. Although these problems are expected to be the most difficult from the test set, TOMLAB / MULTIMIN and TOMLAB / LGO still managed to solve about $40 \%$ of the cases. Comparing Figs. 6 and 10, we observe that the percentage of nonconvex nonsmooth problems solved by several solvers is larger than that for the convex problems. Once again, Table 3 reveals that the nonconvex nonsmooth problems are smaller, on average, than their convex counterparts.

Figures 11 and 12 present the fraction of problems for which each solver found the best solution among all solvers. As seen in these figures, after a brief lead by MCS, TOMLAB / MUL- 


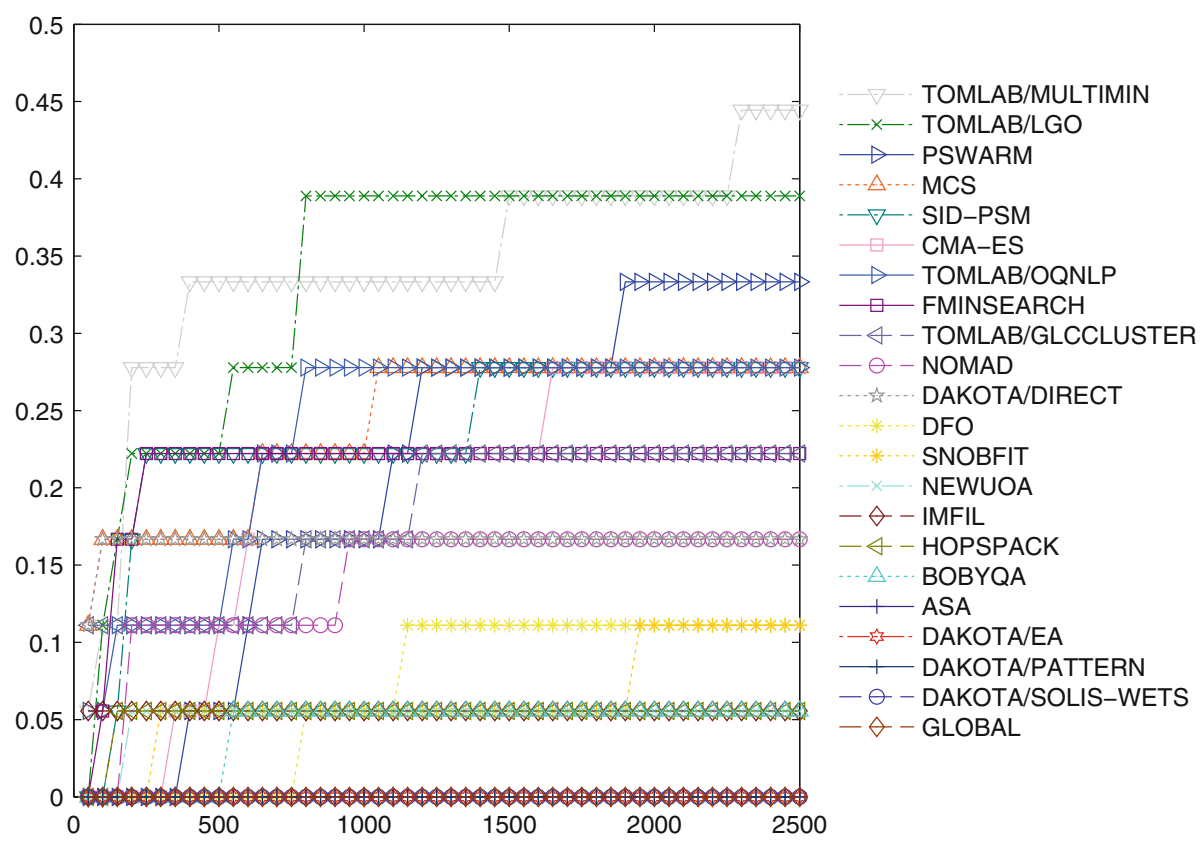

Fig. 10 Fraction of nonconvex nonsmooth problems solved as a function of allowable number of function evaluations

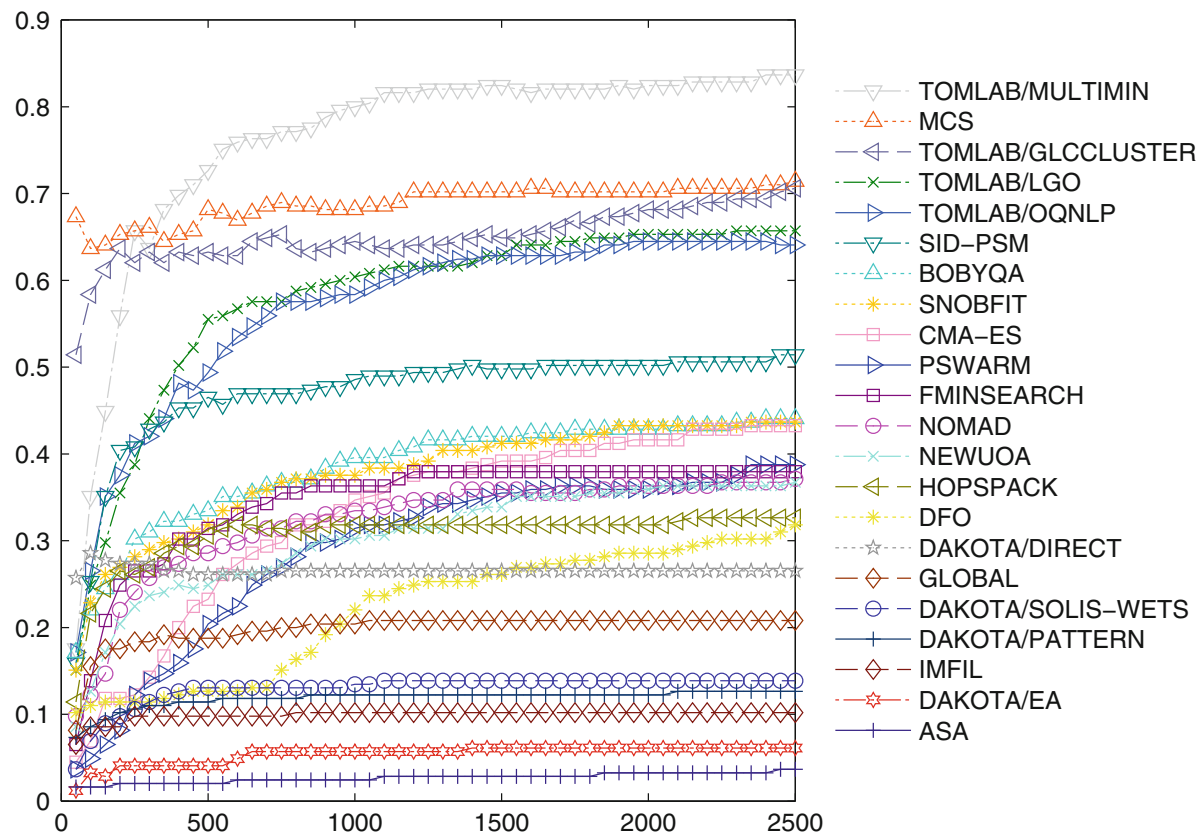

Fig. 11 Fraction of nonconvex smooth problems, as a function of allowable number of function evaluations, for which a solver found the best solution among all solvers tested 


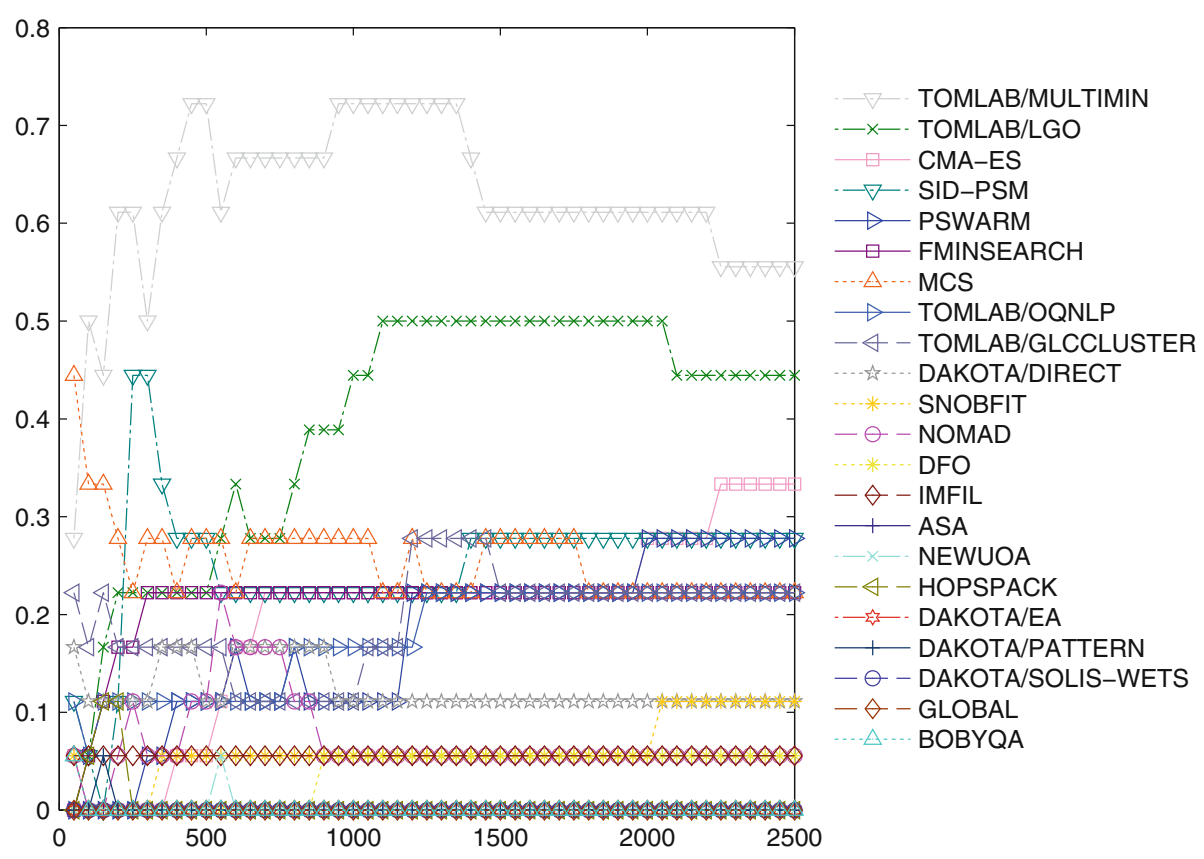

Fig. 12 Fraction of nonconvex nonsmooth problems, as a function of allowable number of function evaluations, for which a solver found the best solution among all solvers tested

TIMIN builds an increasing lead over all other solvers, finding the best solutions for over $83 \%$ of the nonconvex smooth problems. MCS and TOMLAB/GLCCLUSTER follow with $71 \%$. With a final rate of over $56 \%$ of the cases for most of the range, TOMLAB / MULTIMIN is dominant for nonconvex nonsmooth problems.

\subsection{Improvement from starting point}

An alternative benchmark, proposed by Moré and Wild [97], measures each algorithm's ability to improve a starting point. For a given $0 \leq \tau \leq 1$ and starting point $x_{0}$, a solver is considered to have successfully improved the starting point if

$$
f\left(x_{0}\right)-f_{\text {solver }} \geq(1-\tau)\left(f\left(x_{0}\right)-f_{L}\right),
$$

where $f\left(x_{0}\right)$ is the objective value at the starting point, $f_{\text {solver }}$ is the solution reported by the solver, and $f_{L}$ is a lower bound on the best solution identified among all solvers. Since the global solution is known, we used it in place of $f_{L}$ in evaluating this measure. We used this measure to evaluate the average-case performance of each solver, i.e., a problem was considered 'solved' by a solver if the median solution improved the starting point by at least a fraction of $(1-\tau)$ of the largest possible reduction.

Figure 13 presents the fraction of convex smooth problems for which the starting point was improved by a solver as a function of the $\tau$ values. Solvers MCS, DAKOTA/DIRECT, TOMLAB / GLCCLUSTER and TOMLAB / MULTIMIN are found to improve the starting points for $100 \%$ of the problems for $\tau$ values as low as $10^{-6}$. At a first look, it appears pretty remarkable that a large number of solvers can improve the starting point of $90 \%$ of the problems by $90 \%$. Looking more closely at the specific problems at hand reveals that many of them 


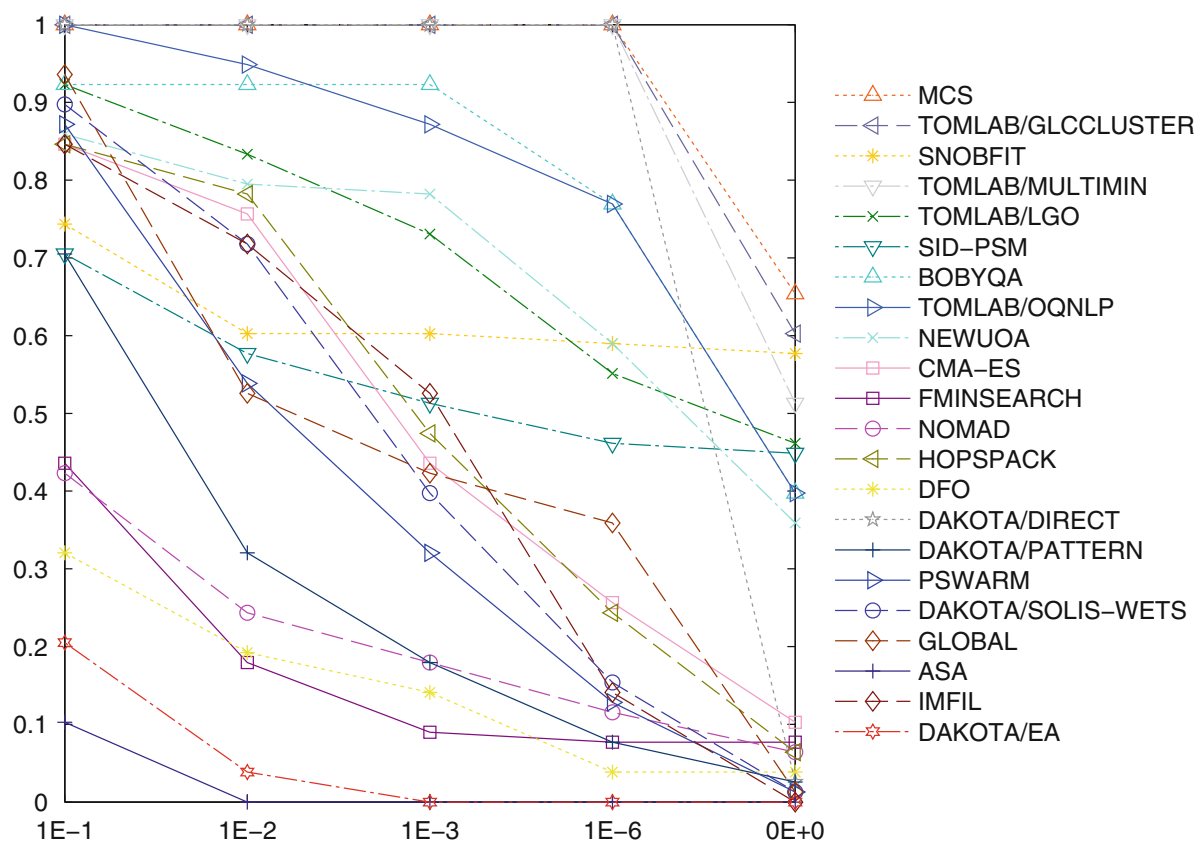

Fig. 13 Fraction of convex smooth problems for which starting points were improved within 2,500 function evaluations vs. $\tau$ values

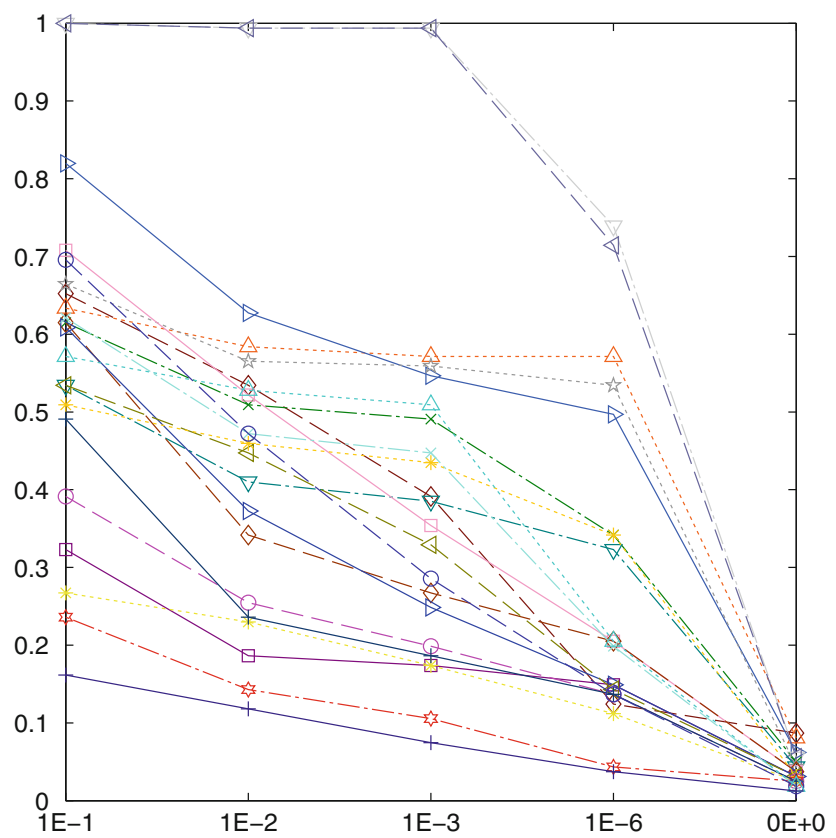

$$
\begin{aligned}
& -\diamond-\text { IMFIL } \\
& \text {-.... MCS } \\
& \triangle \text { TOMLAB/OQNLP } \\
& \text { TOMLAB/MULTIMIN } \\
& -\varangle-\text { TOMLAB/GLCCLUSTER } \\
& \text {-x- TOMLAB/LGO } \\
& -\nabla \text { SID-PSM } \\
& \text {.......... DAKOTA/DIRECT } \\
& \square \text { CMA-ES } \\
& -\vartheta-\text { GLOBAL } \\
& \square \text { FMINSEARCH } \\
& \gg \text { PSWARM } \\
& -\varangle-\text { HOPSPACK } \\
& \text { SNOBFIT } \\
& -\ominus-\text { NOMAD } \\
& \text { DFO } \\
& \text { - DAKOTA/EA } \\
& \text { — DAKOTA/PATTERN } \\
& \text { NEWUOA } \\
& \text { - } \ominus \text { - DAKOTA/SOLIS-WETS } \\
& \text {........ BOBYQA } \\
& \text { ASA }
\end{aligned}
$$

Fig. 14 Fraction of convex nonsmooth problems for which starting points were improved within 2,500 function evaluations vs. $\tau$ values 


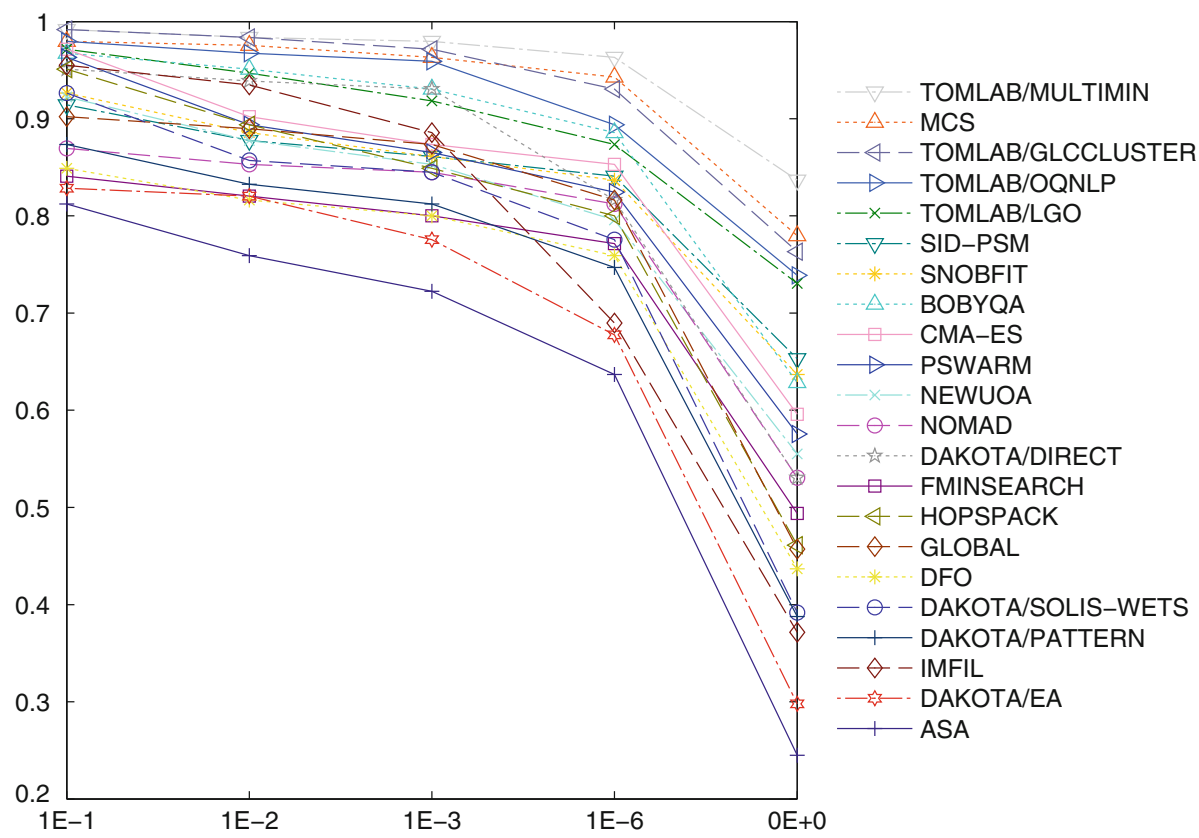

Fig. 15 Fraction of nonconvex smooth problems for which starting points were improved within 2,500 function evaluations vs. $\tau$ values

involve polynomials and exponential terms. As a result, with a bad starting point, the objective function value is easy to improve by $90 \%$, even though the final solution is still far from being optimal. Similarly, Fig. 14 presents the results for convex nonsmooth problems. In comparison with the convex smooth problems, the convex nonsmooth problems display higher percentages and the lines do not drop dramatically. Again, this effect is probably caused by the lower dimensionality (in average) of the nonconvex problems.

Figures 15 and 16 present results for nonconvex problems. As expected, the performance for smooth problems is significantly better than for nonsmooth problems. The performance of MCS, TOMLAB / LGO, TOMLAB / MULTIMIN and TOMLAB / GLCCLUSTER is consistent, at the top group of each of the problem classes.

\subsection{Minimal set of solvers}

Given that no single solver seems to dominate over all others, the next question addressed is whether there exists a minimal cardinality subset of the solvers capable of collectively solving all problems or a certain fraction of all problems. In this case, a problem will be considered solved if any of the chosen solvers succeeds in solving the problem within the optimality tolerance during any one of the ten runs from randomly generated starting points. The results are shown in Figs. 17, 18, 19, and 20 for all combinations of convex/nonconvex and smooth/nonsmooth problems and in Fig. 21 for all problems. For different numbers of function evaluations (vertical axis), the bars in these figures show the fraction of problems (horizontal axis) that are solved by the minimal solver subset. For instance, it is seen in Fig. 17 that one solver (SNOBFIT) is sufficient to solve nearly $13 \%$ of all convex smooth problems 


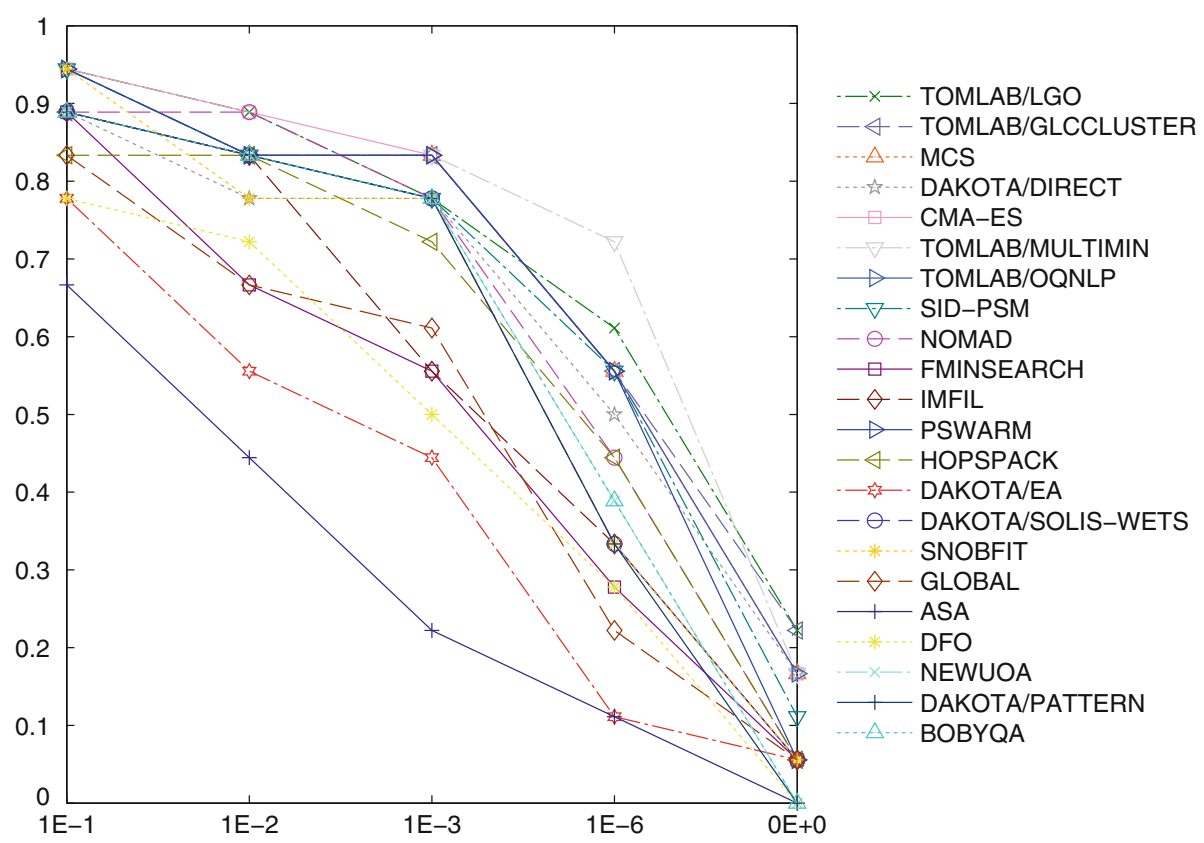

Fig. 16 Fraction of nonconvex nonsmooth problems for which starting points were improved within 2,500 function evaluations vs. $\tau$ values

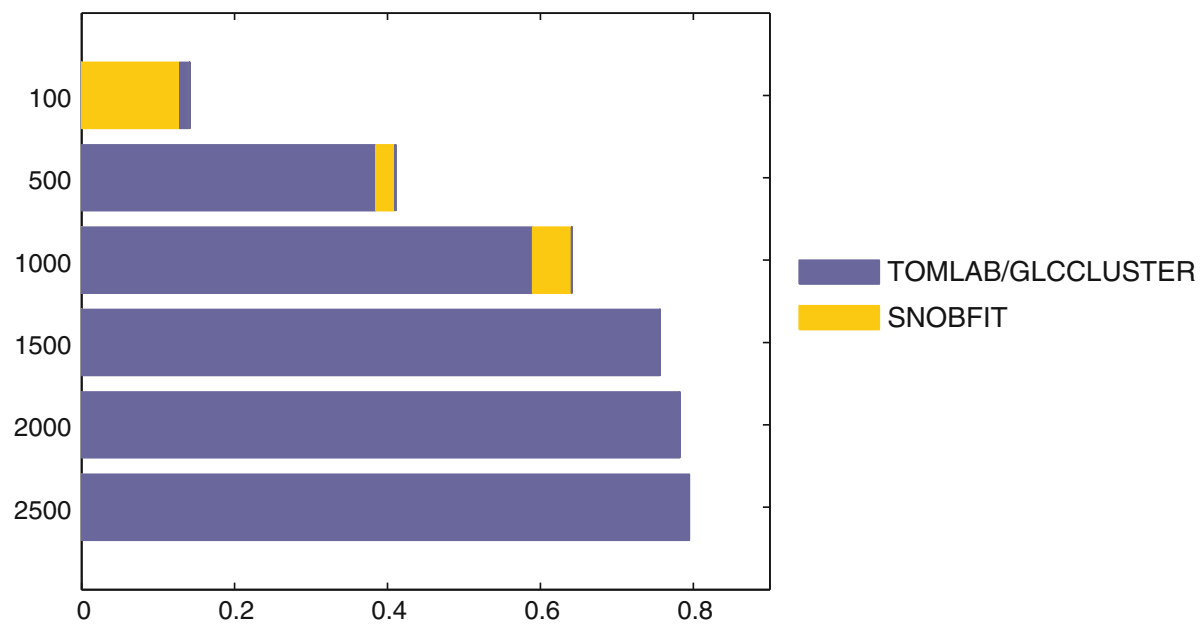

Fig. 17 Minimum number of solvers required to solve convex smooth test problems for various limits of function evaluations (best solver performance)

with 100 function evaluations. The collection of SNOBFIT and TOMLAB / GLCCLUSTER is required to solve up to nearly $15 \%$ of the problems with 100 function evaluations. No other pair of solvers is capable of solving more than $15 \%$ of these problems with 100 function evaluations. Also seen in this figure is that the minimal subset of solvers depends on the number of allowable function evaluations. SNOBFIT and TOMLAB/GLCCLUSTER are in 


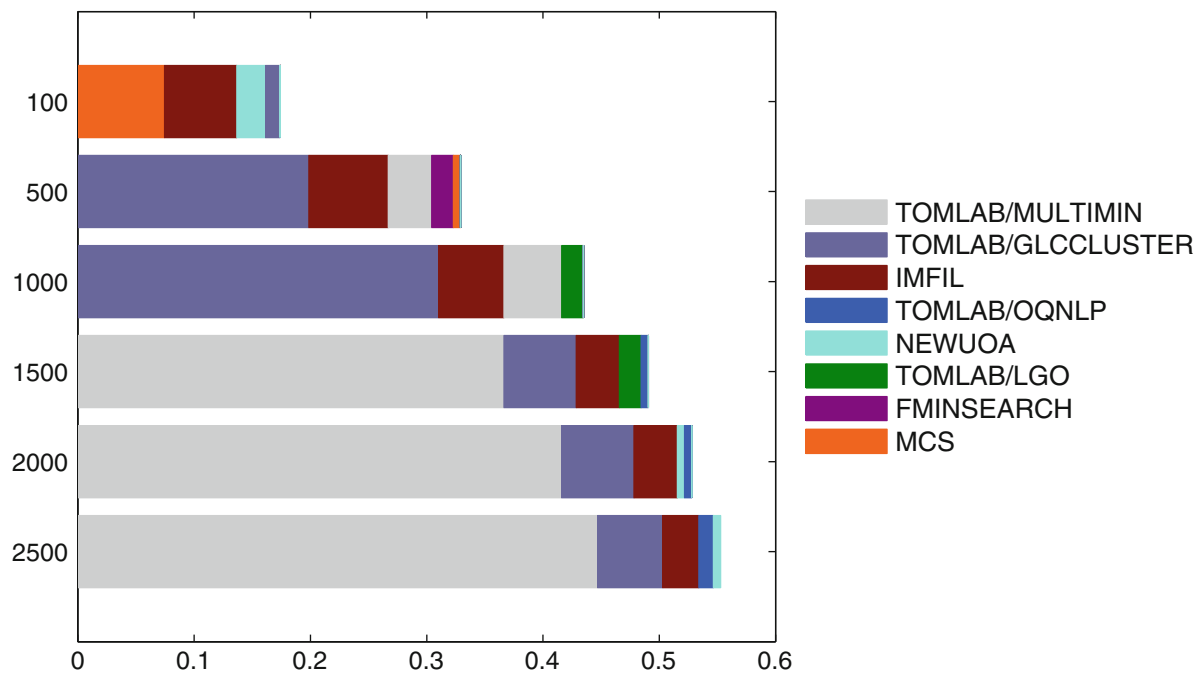

Fig. 18 Minimum number of solvers required to solve convex nonsmooth test problems for various limits of function evaluations (best solver performance)

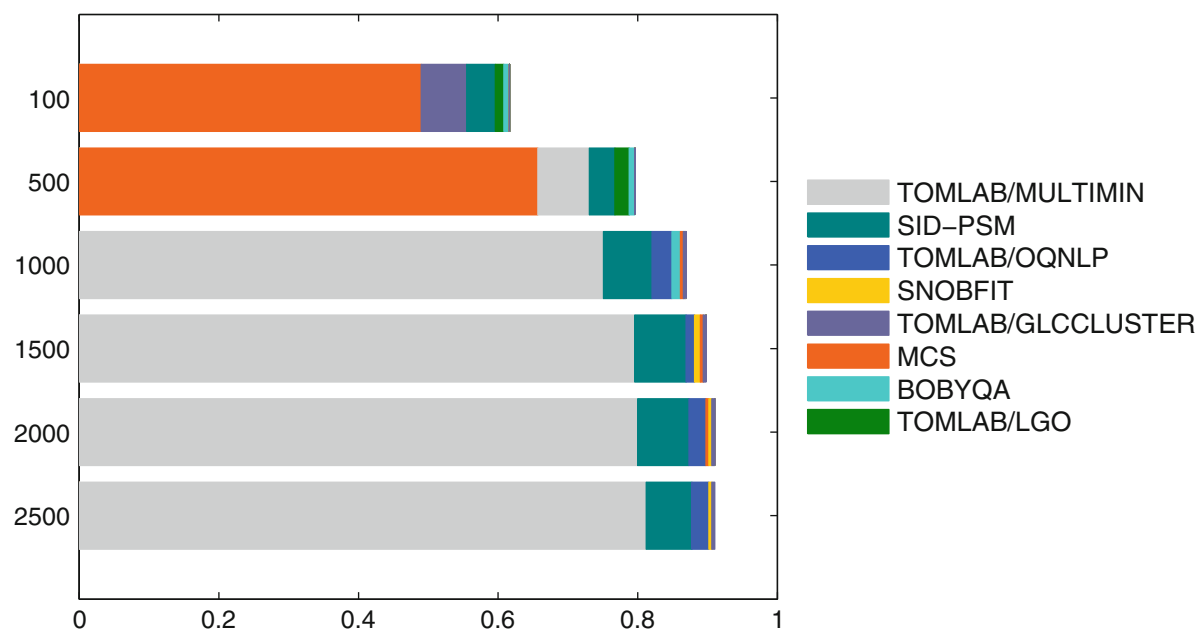

Fig. 19 Minimum number of solvers required to solve nonconvex smooth test problems for various limits of function evaluations (best solver performance)

the minimal set when 1,000 function evaluations are allowed, while SNOBFIT is no longer necessary when more than 1,000 function evaluations are allowed. For convex nonsmooth problems, TOMLAB / MULTIMIN enters the minimal set when 500 or more function evaluations are allowed. TOMLAB / MULTIMIN in combination with TOMLAB/GLCCLUSTER are able to solve over $52 \%$ at 2,500 function evaluations. For nonconvex smooth problems, MCS is found in the minimal set when 500 or less function evaluations are allowed, solving $66 \%$ of the problems at 500 function evaluations. TOMLAB / MULTIMIN is in the minimal set of solvers when 1,000 or more function evaluations are allowed. For nonconvex nonsmooth 


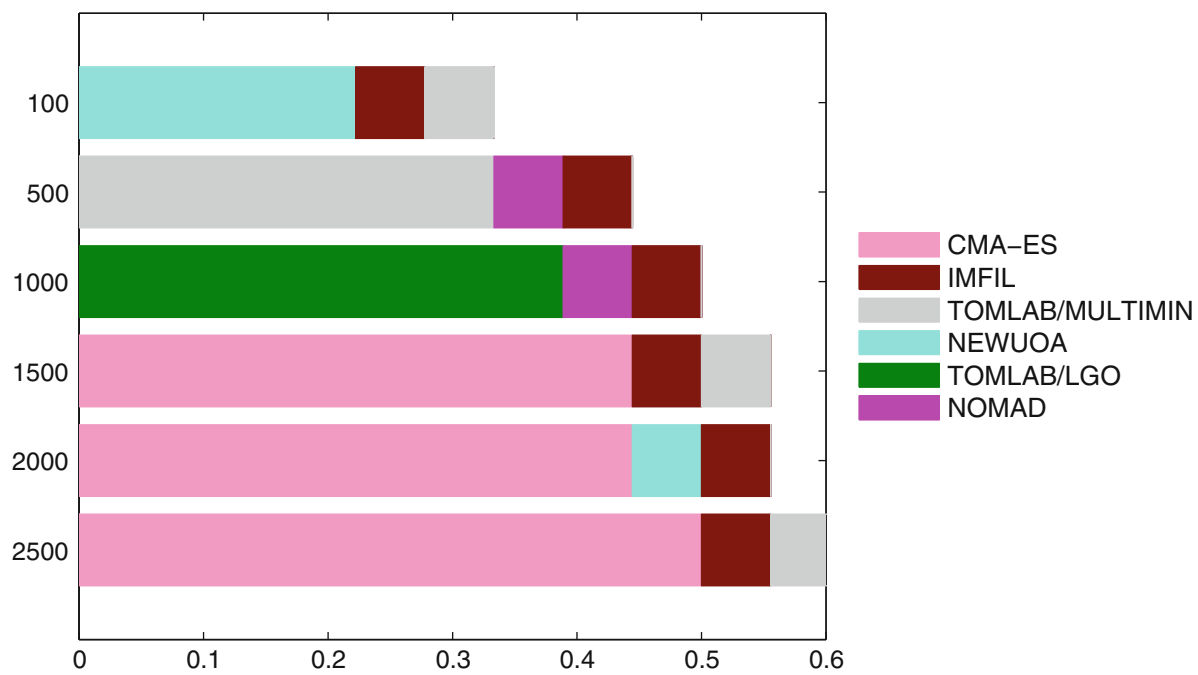

Fig. 20 Minimum number of solvers required to solve nonconvex nonsmooth test problems for various limits of function evaluations (best solver performance)

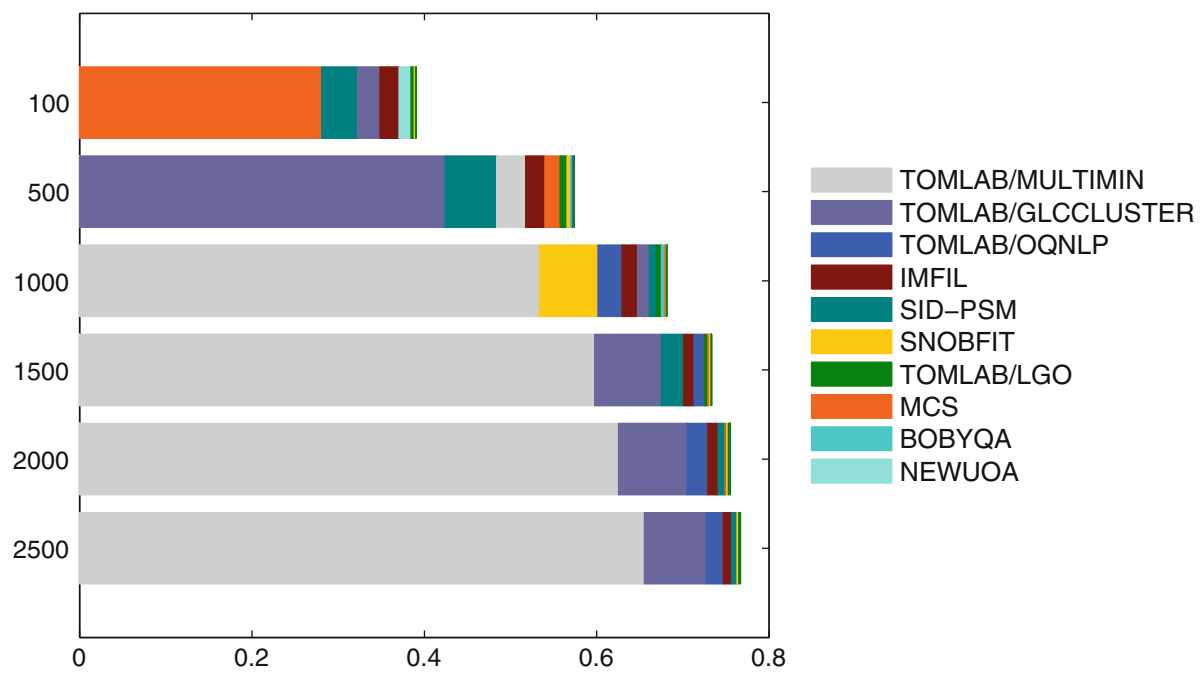

Fig. 21 Minimum number of solvers required to solve all test problems for various limits of function evaluations (best solver performance)

problems, NEWUOA, TOMLAB/MULTIMIN, TOMLAB/LGO, and then CMA-ES solved the largest fraction of problems.

Finally, Fig. 21 shows that TOMLAB / MULTIMIN enters the minimal set of solvers when 500 or more function evaluations are allowed. The combination of TOMLAB / MULTIMIN and TOMLAB / GLCCLUSTER solved the largest fraction of problems over most problem classes. 


\subsection{Impact of problem size}

In general, as the size of the problem increases, the chances of obtaining better solutions clearly decrease. As seen in Fig. 22, over half of the solvers are able to solve $50 \%$ of problems with one or two variables, with TOMLAB / GLCCLUSTER, TOMLAB / MULTIMIN, MCS, TOMLAB / LGO and TOMLAB / OQNLP solving about $90 \%$ of the problems. Figure 23 presents results for problems with three to nine variables. Half of the solvers are able to solve only $29 \%$ of these problems, while TOMLAB/MULTIMIN solves about $78 \%$ of the problems, followed by TOMLAB / LGO, TOMLAB / GLCCLUSTER and MCS.

Results with problems with 10-30 variables are presented in Fig. 24 and show that most of the solvers cannot solve more than $15 \%$ of these problems. Despite the decreasing trend, TOMLAB/MULTIMIN and TOMLAB/GLCCLUSTER are able to solve over $64 \%$ of these problems. Finally, results with problems with 31 to 300 variables are presented in Fig. 25. The same limit of 2,500 function evaluations was used even for the largest problems, in order to test solvers for their ability to adjust to a limited budget of function evaluations. Many of the solvers are not able to solve a single problem, while again TOMLAB / GLCCLUSTER and TOMLAB / MULTIMIN are at the top solving over $28 \%$ of these problems. Once again, a notable degrading performance is displayed by all solvers as problem size increases.

\subsection{Variance of the results}

The previous graphs were presented in terms of median and best results among ten problem instances for each solver with a limit of 2,500 function evaluations. Here, we discuss the variance of these results, as solver performance is dependent on starting point and random

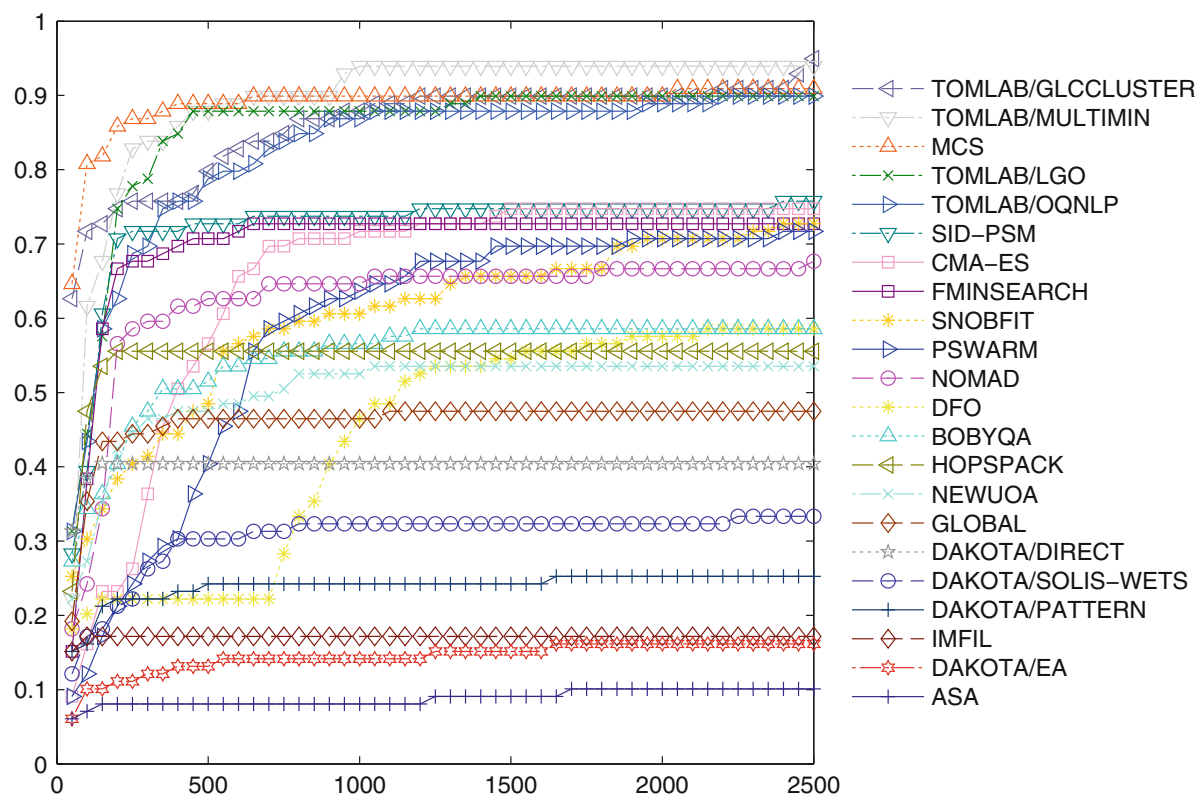

Fig. 22 Fraction of problems with one to two variables that were solved 


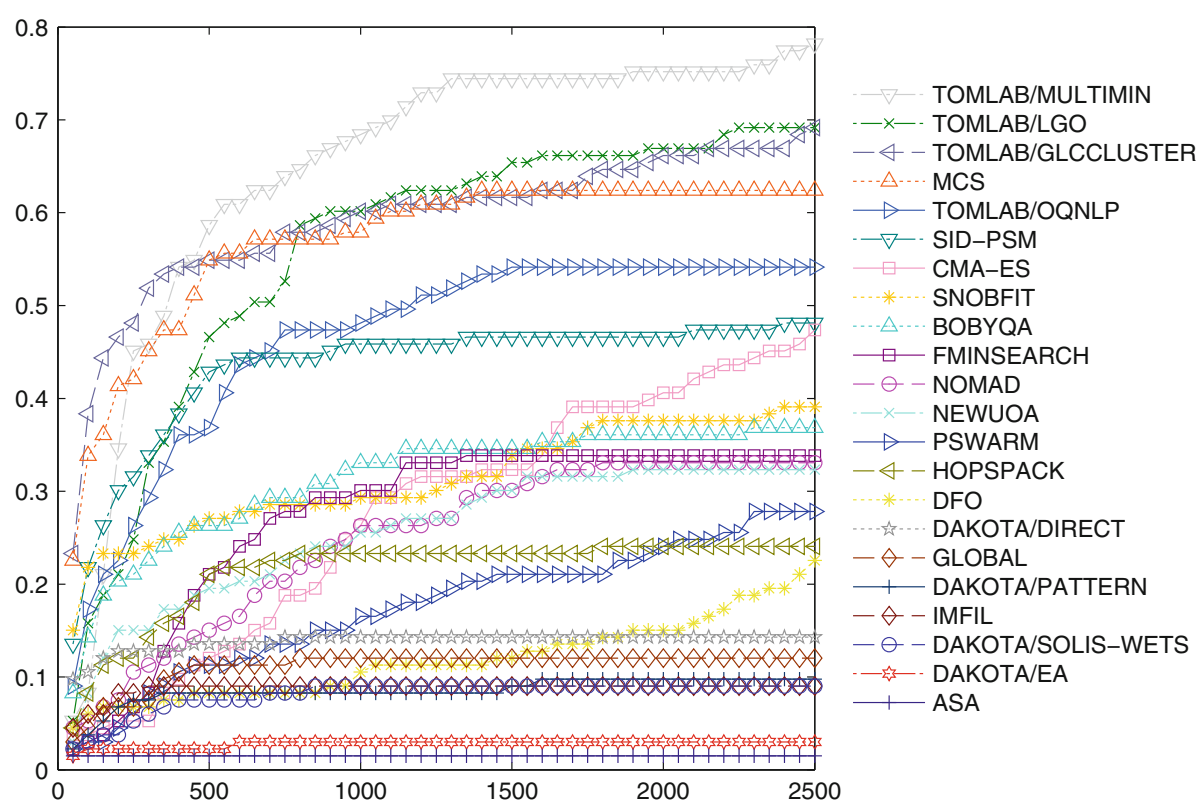

Fig. 23 Fraction of problems with three to nine variables that were solved

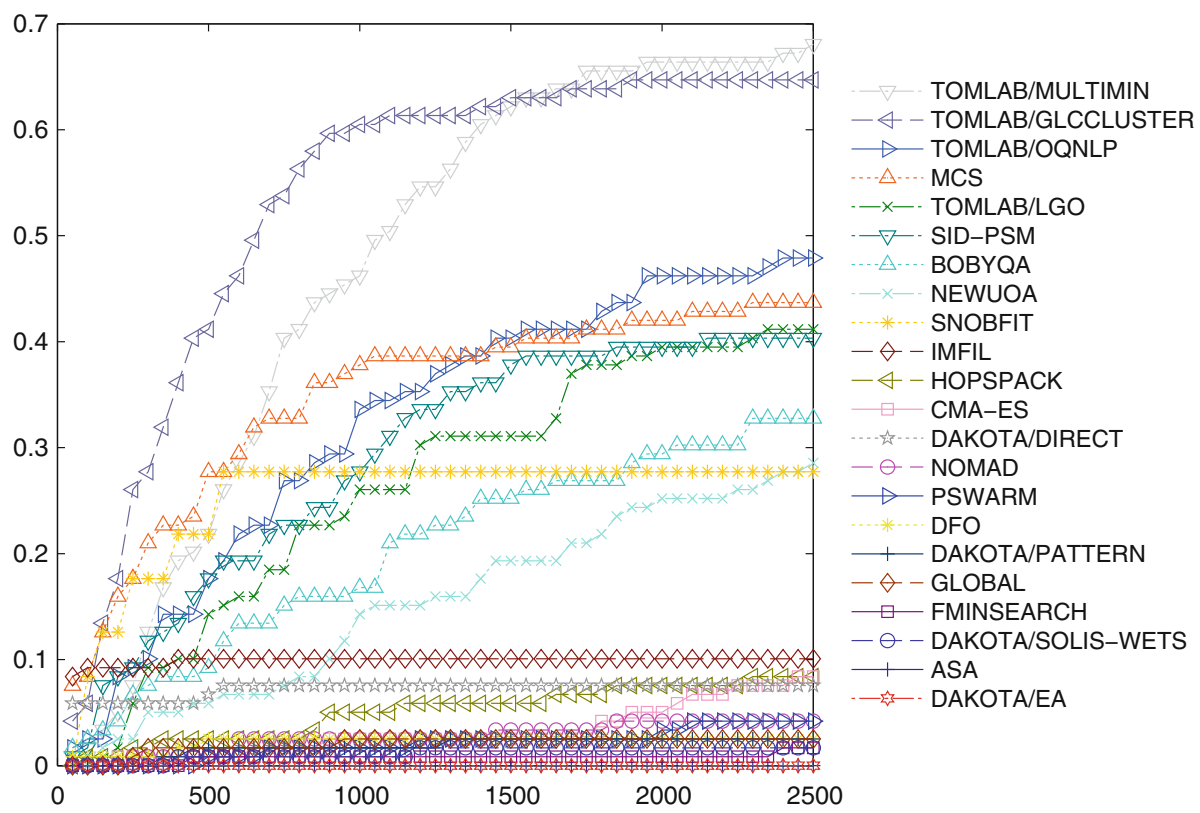

Fig. 24 Fraction of problems with 10-30 variables that were solved

seeds used in the computations. Since the difference in scales of the global solutions and the range of values of the objective function of the test problems prevent a direct comparison, objective function values obtained were scaled as follows: 


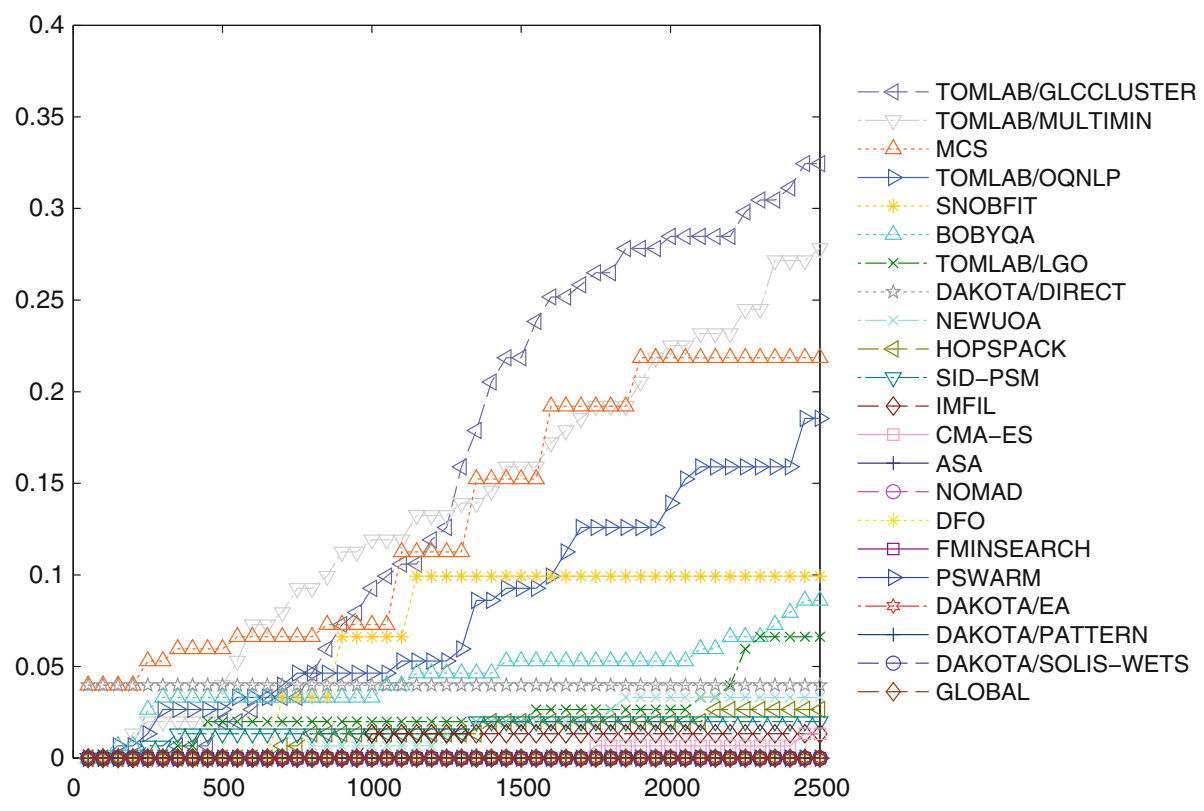

Fig. 25 Fraction of problems with 31-300 variables that were solved

$$
f_{\text {scaled }}=1-\frac{f_{\text {solver }}-f_{L}}{f_{W}-f_{L}},
$$

where $f_{\text {solver }}$ is a solution reported by the solver, $f_{L}$ is the global solution, and $f_{W}$ is the worst solution obtained by the solver among the ten runs from different starting points. The resulting $f_{\text {scaled }}$ is in the interval $[0,1]$ with a value of 1 corresponding to the global solution and a value of 0 corresponding to the worst solution reported by the solver.

Figure 26 displays the average scaled best, mean, median and worst results among the ten optimization instances for all test problems. TOMLAB / GLCCLUSTER and TOMLAB / MULTIMIN achieve results close to 1 and with low variability among the best, mean, median and worst results. Detailed variability graphs are presented for each solver in the on-line material.

\subsection{Impact of missing bounds}

As presented in Table 4, a significant number of test problems contain at least one variable without lower or upper bounds. Figure 27 presents the fraction of problems solved for problems grouped by the availability of bounds on their variables. Problems with bounds on all their variables display a much higher percentage of success than the other two classes. Better results are obtained for entirely unbounded problems compared to problems with only lower bounds available. This is caused by the corresponding problem sizes (see Table 4). CMA-ES, PSWARM, NOMAD and GLOBAL are found to be most sensitive to the absence of bounds.

\subsection{Refinement ability}

The following experiment was designed to analyze the ability of solvers to obtain a highly accurate solution. The solvers were provided an initial solution close to a global solution of 


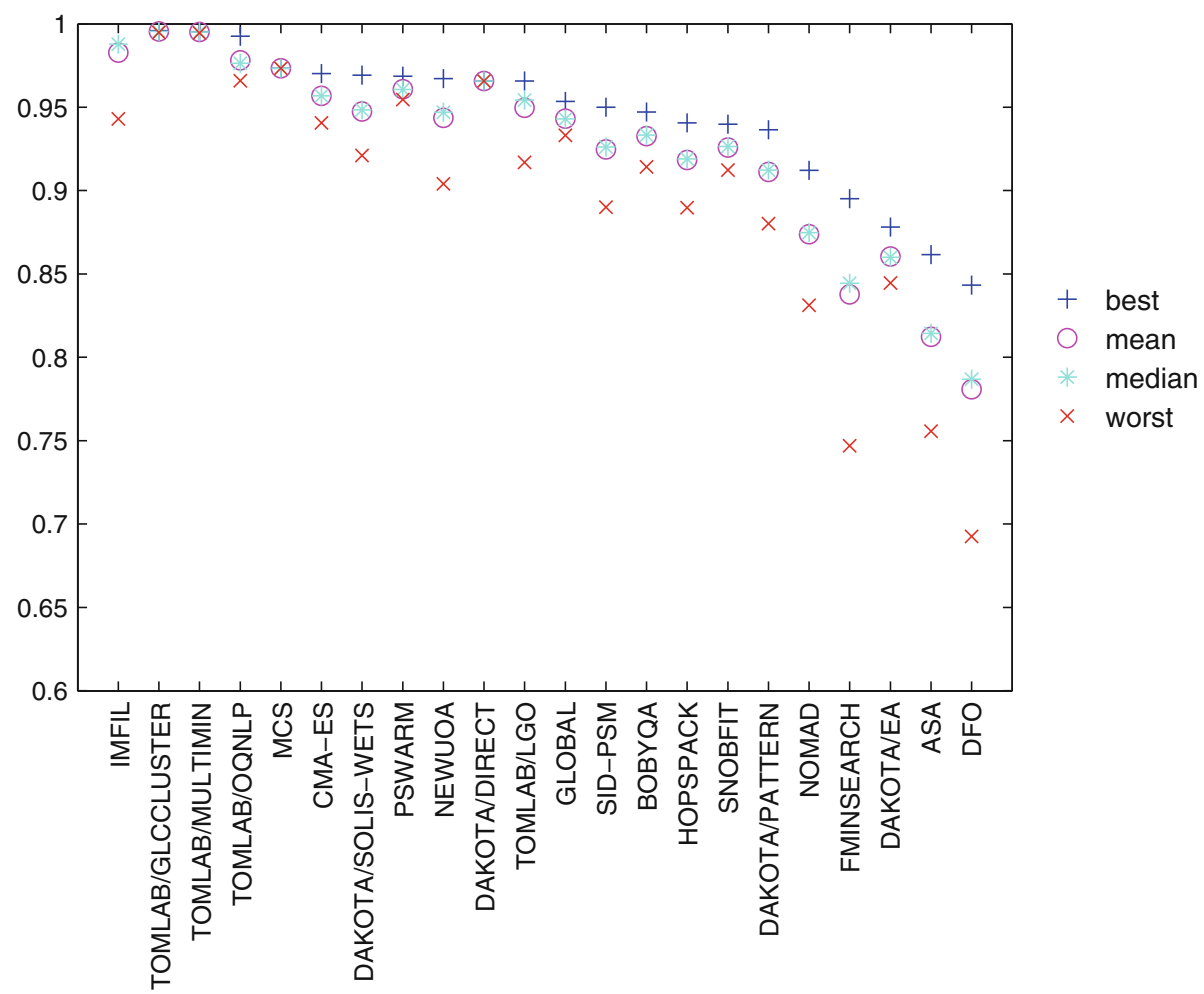

Fig. 26 Scaled results for the best, mean, median and worst result among the 10 optimization instances after 2,500 function evaluations for each solver

the problem and a feasible space that was asymmetric with respect to the global solution $x^{*}$. In particular, the ranges of all variables were set to $\left[-0.166+x^{*}, 0.033+x^{*}\right]$, allowing each variable a range of 0.2 , unless the original problem bounds were tighter, in which case the latter were used.

Figure 28 presents the fraction of problems of different size that were solved to within the optimality tolerance. It can be noted that for problems with one or two variables, most of the solvers are able to find the global solution in over $90 \%$ of the cases. For problems with three to nine variables, only 8 solvers are able to solve more than $90 \%$ of the cases. TOMLAB / GLCCLUSTER solves most problems involving ten to thirty variables, with just over $71 \%$ of the problems, followed within $5 \%$ by five other solvers. Performance for problems with over thirty variables drops significantly for all solvers except for TOMLAB / OQNLP, NEWUOA and BOBYQA, which solve around 50\% of the problems. Figs. 29, 30, 31, and 32 present similar refinement ability results for each problem class, while the on-line supplement presents detailed graphs for each solver separately.

\subsection{Solution of a test set unseen by developers}

In an effort to encourage developers to improve their implementations, the above computational results were shared with developers over several rounds in the course of our study. The developers of DAKOTA, IMFIL, SID-PSM, and the TOMLAB solvers improved their codes 

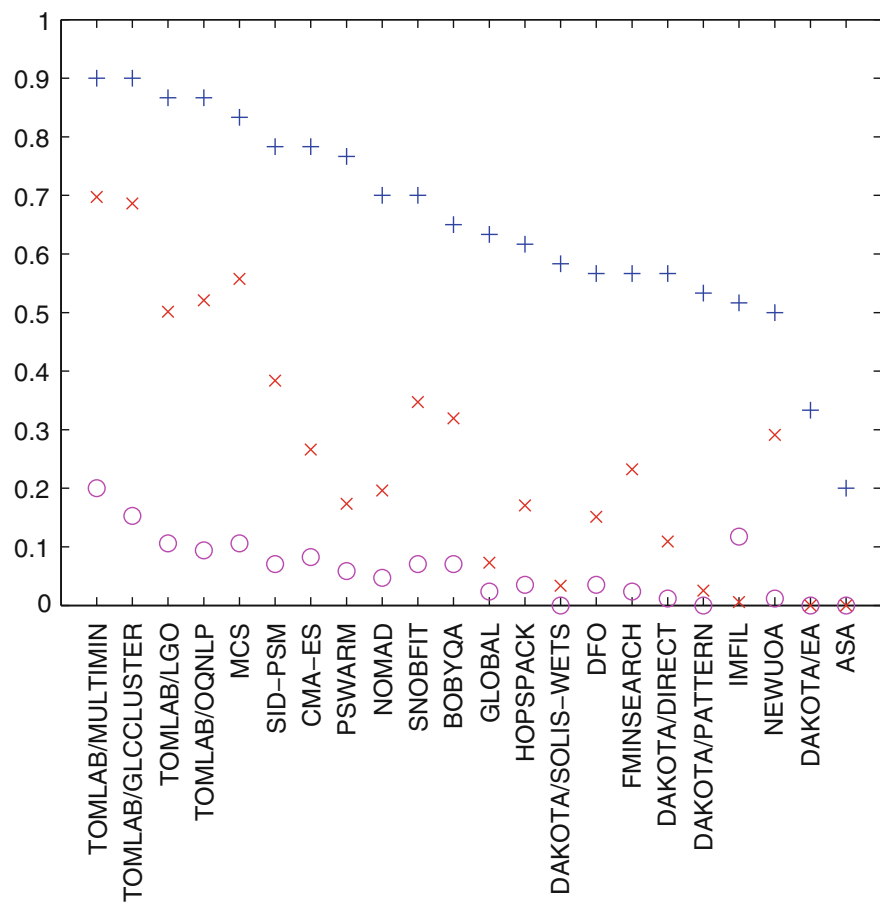

Fig. 27 Fraction of problems solved after 2,500 function evaluations for classes of problems by availability of bounds on their variables

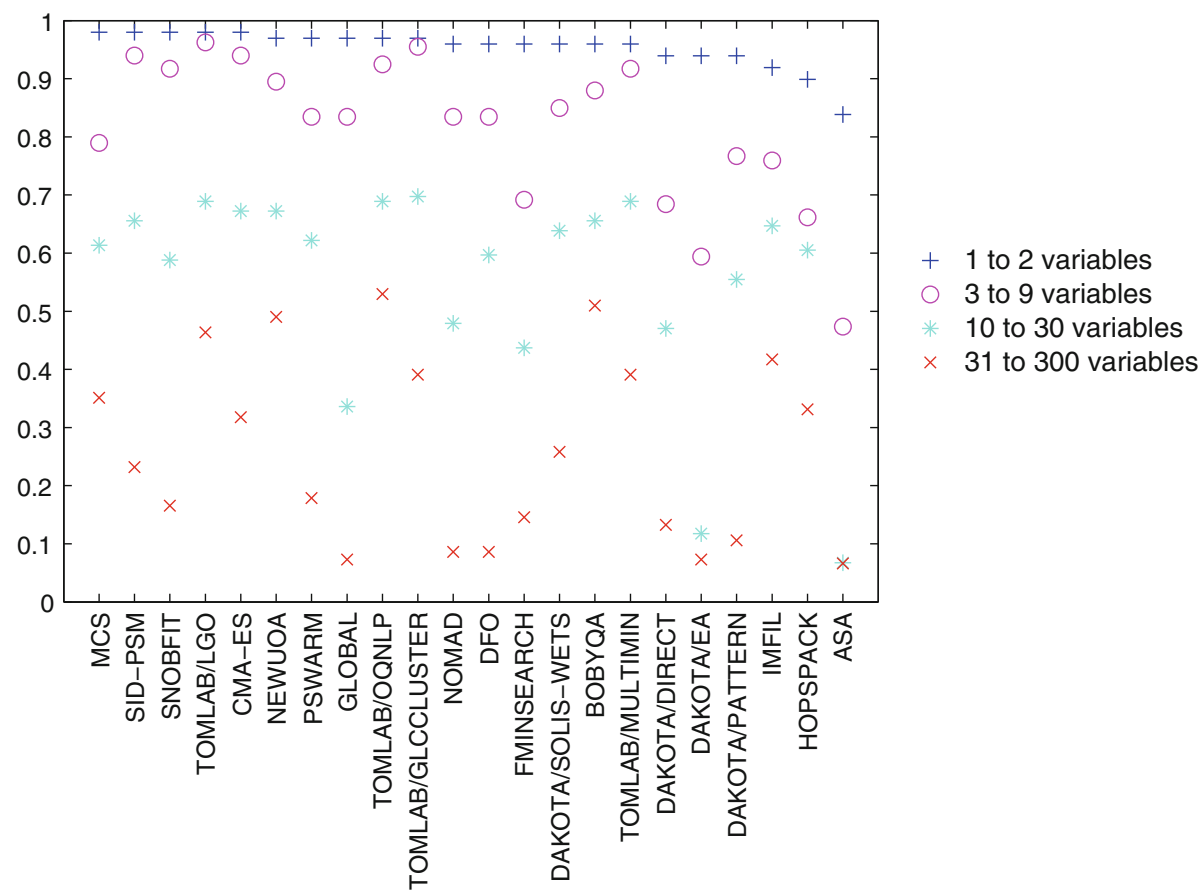

Fig. 28 Fraction of problems solved from a near-optimal solution 


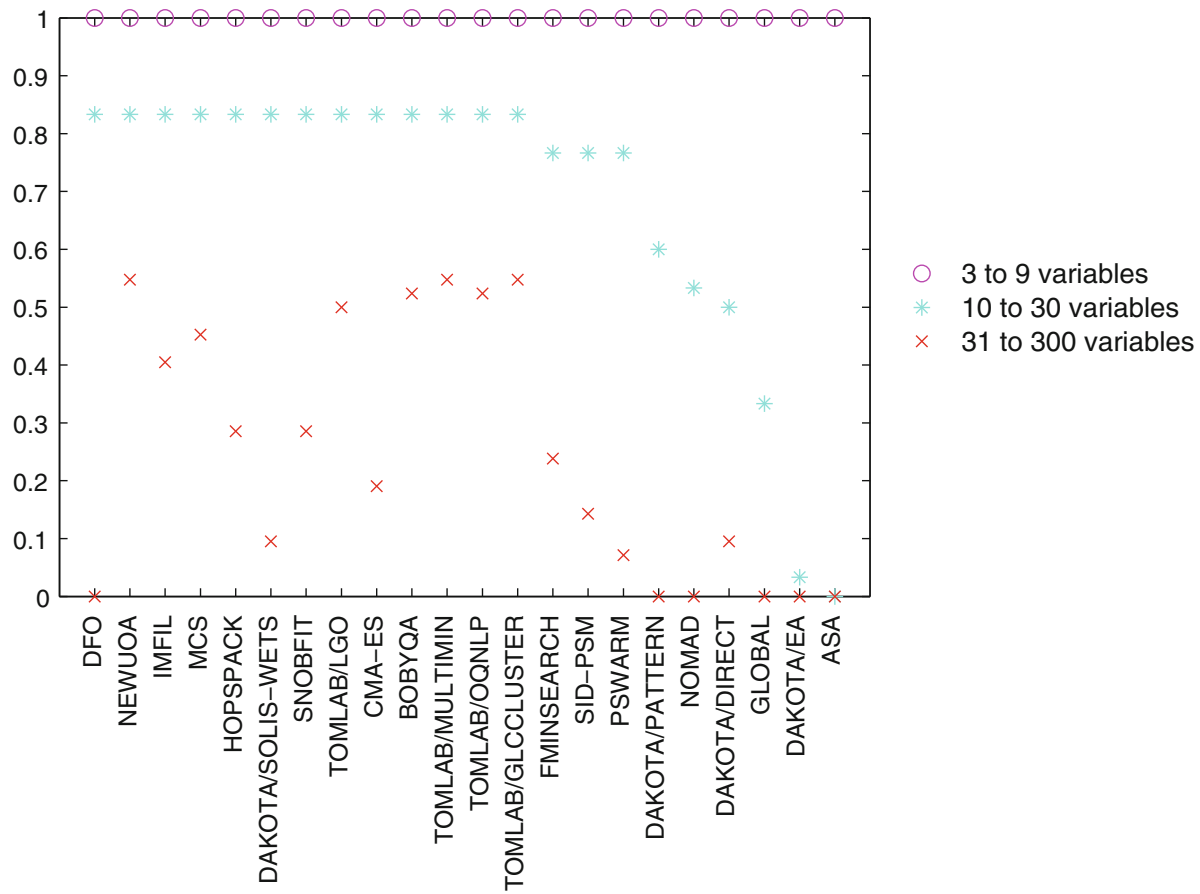

Fig. 29 Fraction of convex smooth problems solved from a near-optimal solution

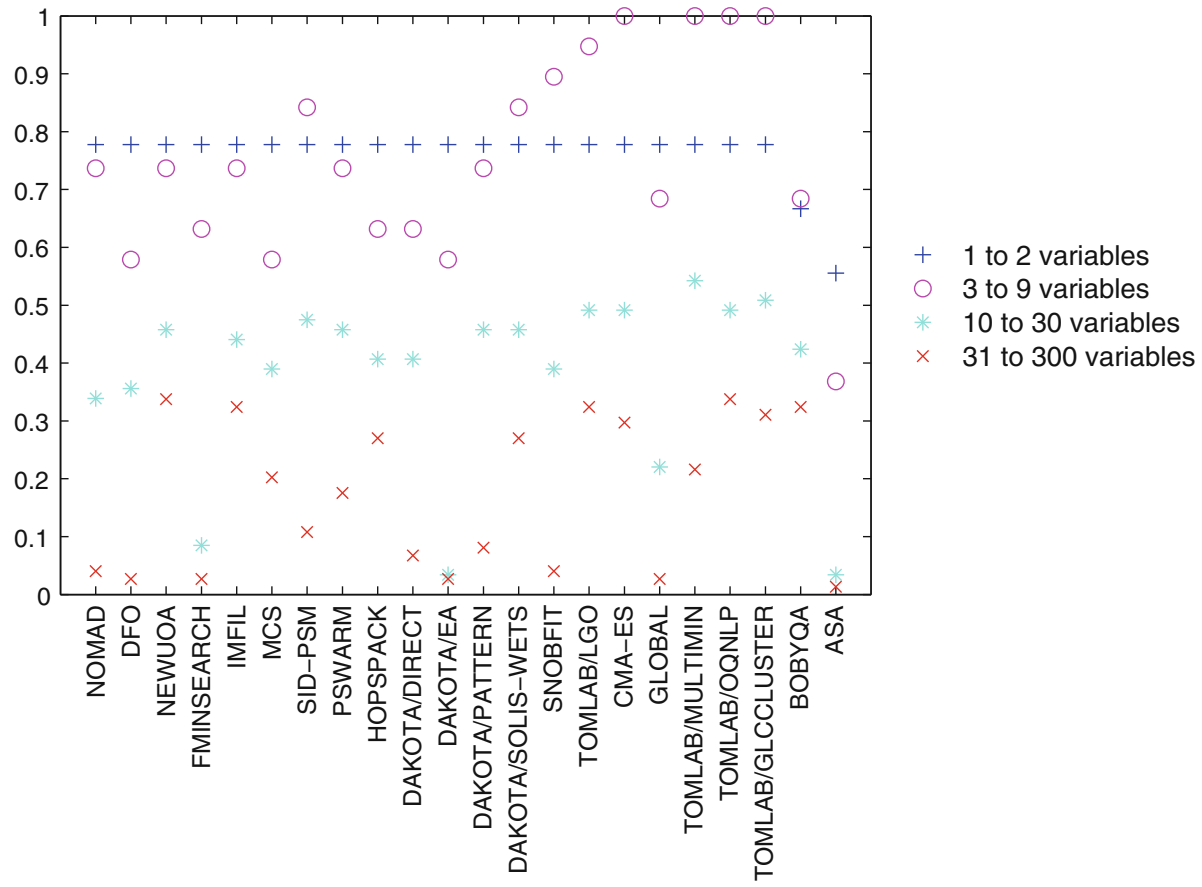

Fig. 30 Fraction of convex nonsmooth problems solved from a near-optimal solution 


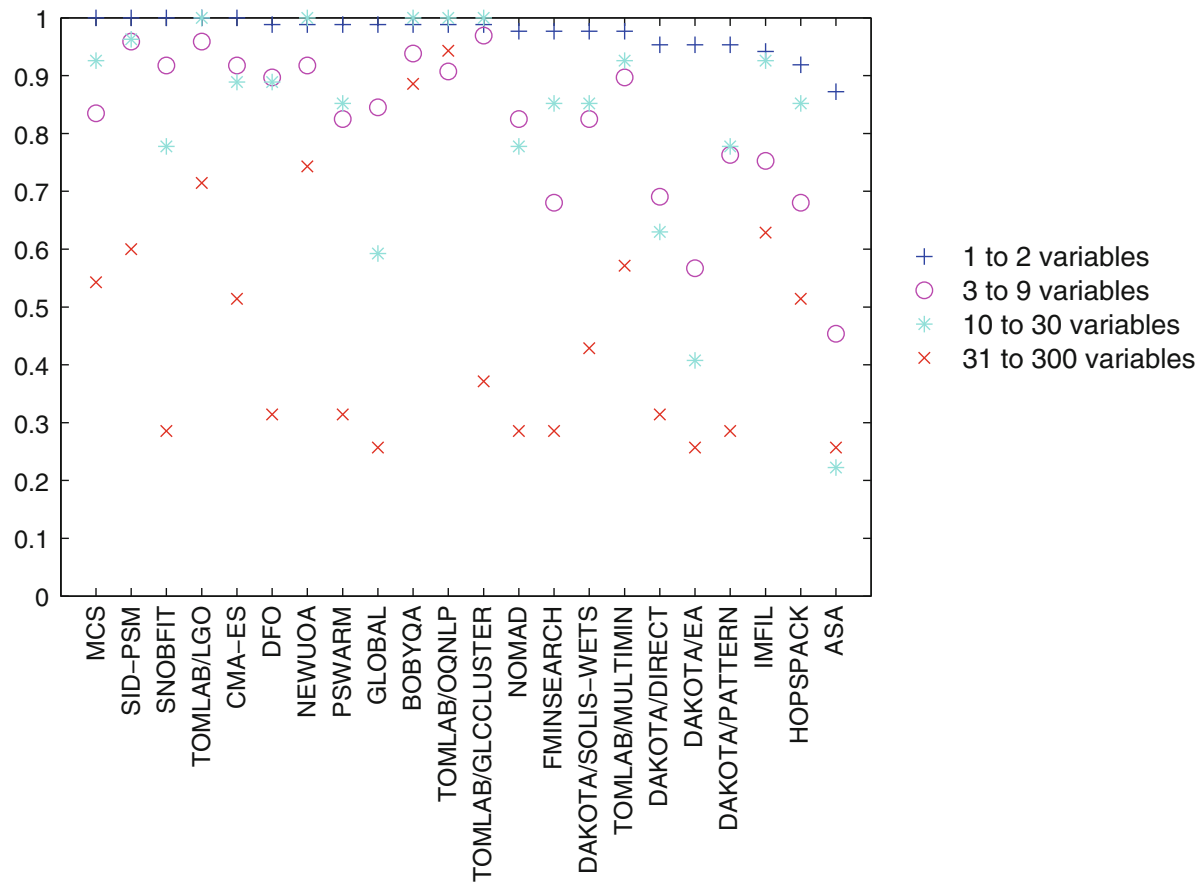

Fig. 31 Fraction of nonconvex smooth problems solved from a near-optimal solution

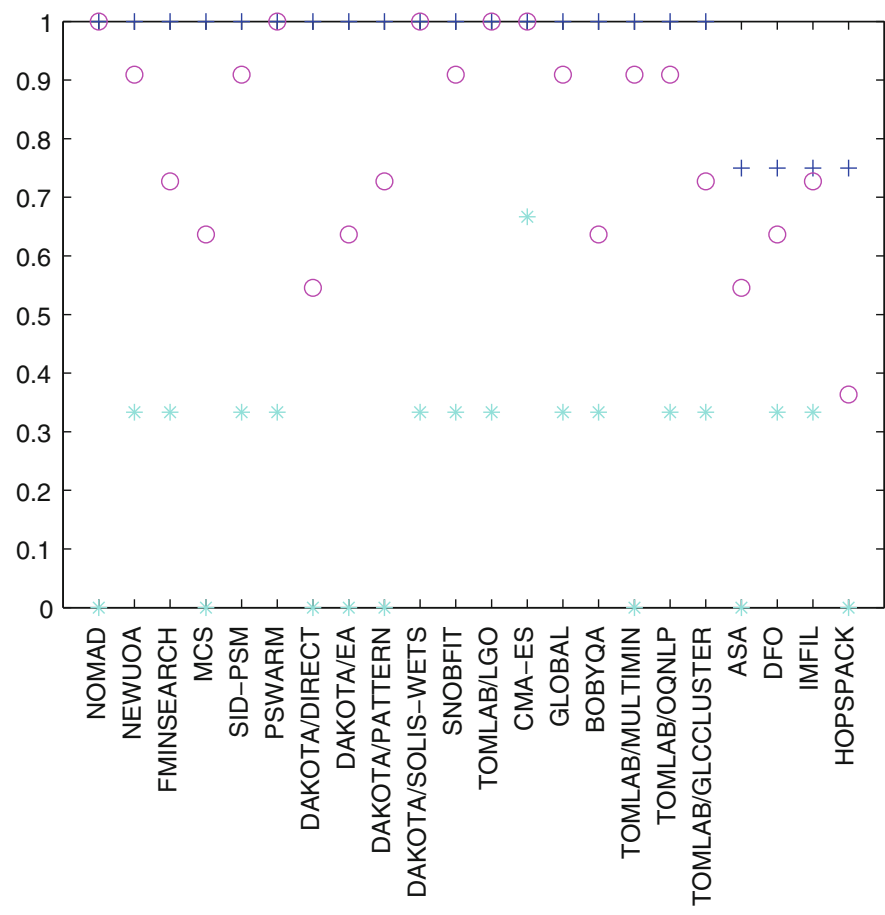

Fig. 32 Fraction of nonconvex nonsmooth problems solved from a near-optimal solution

+1 to 2 variables

3 to 9 variables 10 to 30 variables 
using feedback from our results. This raises the question whether this process may have led to overtraining of the software on our test problem collection. To address this question, we solved a set of an additional 502 problems that had never been seen by the developers. These additional test problems were obtained from the original ones via a transformation of variables that preserves smoothness and convexity characteristics but otherwise changes the problems considerably, including the shape of the objective functions and location of all local solutions. In particular, the following linear transformation was used:

$$
\begin{array}{r}
\bar{x}=x+\left(1+t u^{\prime} x\right) u, \\
t=(c-1) / u^{\prime} u,
\end{array}
$$

where $u$ is a randomly generated vector with elements in $[0,1)$, and $c$ is the condition number of the transformation. We chose $c=2$, so as to result in a variable space that is of similar size to that of the original space. The results with and without the transformation were almost identical.

\section{Conclusions}

While much work is still to be done, especially for the constrained case, significant progress has been made on the algorithmic and theoretical aspects of derivative-free optimization over the past two decades. Without doubt, the most important results from this activity are the recent model-based algorithms as well as proofs of global convergence for direct and model-based approaches.

A set of 22 leading software implementations of state-of-the-art derivative-free optimization algorithms were tested on a large collection of publicly available problems, whose solutions were obtained using derivative-based and global optimization algorithms. Our computational results show that attaining the best solutions even for small problems is a challenge for most current derivative-free solvers. The solvers TOMLAB / MULTIMIN, TOMLAB / GLCCLUSTER, MCS and TOMLAB/LGO, on average, provide the best solutions among all the solvers tested. However, computational results show that there is no single solver whose performance dominates that of all others. In addition, all solvers provided the best solution possible for at least some of the test problems. Although no subset of solvers suffices to solve all problems, our results suggest that the combination of the commercial TOMLAB / MULTIMIN and TOMLAB/GLCCLUSTER with the free MCS and SNOBFIT is sufficient to provide the best results in most cases. Problem dimensionality and nonsmoothness were found to rapidly increase the complexity of the search and decrease performance for all solvers. Finally, from a starting point close to a solution, TOMLAB / OQNLP, NEWUOA and TOMLAB / MULTIMIN showed the fastest convergence towards the solution. Missing bounds on the variables are found to affect significantly the performance of all solvers, particularly the stochastic ones.

The issues of explicit or hidden constraints and noise in the objective function calculation have not been addressed in this paper. These issues are complex and warrant further study on their own. In this direction, we performed experiments with applications for which the objective function was a true black-box that was not available in algebraic form. The results from these experiments suggest that the solvers identified as best herein indeed suffice to address a variety of true black-box application problems. These results are detailed elsewhere $[13,26,43,120,128,139,144]$. 
Acknowledgments This work was supported over a period of seven years by the Joint NSF/NIGMS Initiative to Support Research in the Area of Mathematical Biology under NIH award GM072023, National Energy Technology Laboratory's on-going research in $\mathrm{CO}_{2}$ capture under the RES contract DE-FE-0004000, and the National Science Foundation under award CBET-1033661. The quality of this paper benefited significantly from discussions with the authors of the software listed in Table 2. Their constructive comments and suggestions on several drafts of this paper are far too many to be acknowledged individually.

\section{References}

1. Aarts, E.H.L., van Laarhoven, P.J.M.: Statistical cooling: a general approach to combinatorial optimization problems. Phillips J. Res. 40, 193-226 (1985)

2. Abramson, M.A.: Pattern Search Algorithms for Mixed Variable General Constrained Optimization Problems. PhD thesis, Department of Computational and Applied Mathematics, Rice University, Houston (2002, Aug)

3. Abramson, M.A.: NOMADm Version 4.5 User's Guide. Air Force Institute of Technology, Wright-Patterson AFB, OH (2007)

4. Abramson, M.A., Asaki, T.J., Dennis, J.E. Jr., O’Reilly, K.R., Pingel, R.L.: Quantitative object reconstruction via Abel-based X-ray tomography and mixed variable optimization. SIAM J. Imaging Sci. 1, 322-342 (2008)

5. Abramson, M.A., Audet, C.: Convergence of mesh adaptive direct search to second-order stationary points. SIAM J. Optim. 17, 606-609 (2006)

6. Abramson, M.A., Audet, C., Couture, G., Dennis, J.E. Jr., LeDigabel, S.: The NomaD project. http:// www.gerad.ca/nomad/

7. Abramson, M.A., Audet, C., Dennis, J.E. Jr.: Filter pattern search algorithms for mixed variable constrained optimization problems. Pac. J. Optim. 3, 477-500 (2007)

8. Abramson, M.A., Audet, C., Dennis, J.E. Jr., Le Digabel, S.: OrthoMADS: a deterministic MADS instance with orthogonal directions. SIAM J. Optim. 20, 948-966 (2009)

9. Audet, C.: Convergence results for generalized pattern search algorithms are tight. Optim. Eng. 5, 101122 (2004)

10. Audet, C., Béchard, V., Chaouki, J.: Spent potliner treatment process optimization using a MADS algorithm. Optim. Eng. 9, 143-160 (2008)

11. Audet, C., Dennis, J.E. Jr.: Mesh adaptive direct search algorithms for constrained optimization. SIAM J. Optim. 17, 188-217 (2006)

12. Audet, C., Dennis, J.E. Jr.: A progressive barrier for derivative-free nonlinear programming. SIAM J. Optim. 20, 445-472 (2009)

13. Awasthi, S.: Molecular Docking by Derivative-Free Optimization Solver. Master's thesis, Department of Chemical Engineering, Carnegie Mellon University, Pittsburgh (2008)

14. Barros, P.A. Jr., Kirby, M.R., Mavris, D.N.: Impact of sampling techniques selection on the creation of response surface models. SAE Trans. J. Aerosp. 113, 1682-1693 (2004)

15. Bartholomew-Biggs, M.C., Parkhurst, S.C., Wilson, S.P.: Using DIRECT to solve an aircraft routing problem. Comput. Optim. Appl. 21, 311-323 (2002)

16. Barton, R.R.: Metamodeling: A state of the art review. In: Proceedings of the 1994 Winter Simulation Conference, pp. 237-244 (1994)

17. Bélisle, C.J., Romeijn, H.E., Smith, R.L.: Hit-and-run algorithms for generating multivariate distributions. Math. Oper. Res. 18, 255-266 (1993)

18. Bethke, J.D.: Genetic Algorithms as Function Optimizers. PhD thesis, Department of Computer and Communication Sciences, University of Michigan, Ann Arbor (1980)

19. Björkman, M., Holmström, K.: Global optimization of costly nonconvex functions using radial basis functions. Optim. Eng. 1, 373-397 (2000)

20. Boender, C.G.E., Rinnooy Kan, A.H.G., Timmer, G.T.: A stochastic method for global optimization. Math. Program. 22, 125-140 (1982)

21. Boneh, A., Golan, A.: Constraints' redundancy and feasible region boundedness by random feasible point generator (RFPG). In: 3rd European Congress on Operations Research (EURO III), Amsterdam (1979)

22. Booker, A.J., Dennis, J.E., Jr., Frank, P.D., Serafini, D.B., Torczon, V.J., Trosset, M.W.: A rigorous framework for optimization of expensive functions by surrogates. In: ICASE Report, pp. 1-24 (1998)

23. Booker, A.J., Dennis, J.E. Jr., Frank, P.D., Serafini, D.B., Torczon, V.J., Trosset, M.W.: A rigorous framework for optimization of expensive functions by surrogates. Struct. Optim. 17, 1-13 (1999) 
24. Booker, A.J., Meckesheimer, M., Torng, T.: Reliability based design optimization using design explorer. Optim. Eng. 5, 179-205 (2004)

25. Brent, R.P.: Algorithms for Minimization without Derivatives. Prentice-Hall, Englewood Cliffs (1973)

26. Chang, K.-F.: Modeling and Optimization of Polymerase Chain Reaction Using Derivative-Free Optimization. Master's thesis, Department of Chemical Engineering, Carnegie Mellon University, Pittsburgh (2011)

27. Chiang, T., Chow, Y.: A limit theorem for a class of inhomogeneous Markov processes. Ann. Probab. 17, 1483-1502 (1989)

28. COIN-OR Project. Derivative Free Optimization. http://projects.coin-or.org/Dfo

29. COIN-OR Project. IPOPT 2.3.x A software package for large-scale nonlinear optimization. http:// www.coin-or.org/Ipopt/ipopt-fortran.html

30. Conn, A.R., Gould, N., Lescrenier, M., Toint, Ph.L.: Performance of a multifrontal scheme for partially separable optimization. In: Gomez, S., Hennart, J.-P. (eds.) Advances in Optimization and Numerical Analysis, pp. 79-96. Kluwer, Dordrecht (1994)

31. Conn, A.R., Scheinberg, K., Toint, P.L. : On the convergence of derivative-free methods for unconstrained optimization. In: Buhmann, M.D., Iserles, A. (eds.) Approximation Theory and Optimization, Tribute to M. J. D. Powell, pp. 83-108. Cambridge University Press, Cambridge (1996)

32. Conn, A.R., Scheinberg, K., Toint, P.L.: Recent progress in unconstrained nonlinear optimization without derivatives. Math. Program. 79, 397-414 (1997)

33. Conn, A.R., Scheinberg, K., Toint, P.L.: A derivative free optimization algorithm in practice. In: Proceedings of AIAA St Louis Conference, pp. 1-11 (1998)

34. Conn, A.R., Scheinberg, K., Vicente, L.N.: Global convergence of general derivative-free trust-region algorithms to first and second order critical points. SIAM J. Optim. 20, 387-415 (2009)

35. Conn, A.R., Scheinberg, K., Vicente, L.N.: Introduction to derivative-free optimization. SIAM, Philadelphia (2009)

36. Cox, D.D., John, S.: SDO: A statistical method for global optimization. In: Multidisciplinary Design Optimization (Hampton, VA, 1995), pp. 315-329. SIAM, Philadelphia (1997)

37. Csendes, T., Pál, L., Sendín, J.O.H., Banga, J.R.: The GLOBAL optimization method revisited. Optim. Lett. 2, 445-454 (2008)

38. Custódio, A.L., Dennis, J.E. Jr., Vicente, L.N.: Using simplex gradients of nonsmooth functions in direct search methods. IMA J. Numer. Anal. 28, 770-784 (2008)

39. Custódio, A.L., Rocha, H., Vicente, L.N.: Incorporating minimum Frobenius norm models in direct search. Comput. Optim. Appl. (to appear)

40. Custódio, A.L., Vicente, L.N.: Using sampling and simplex derivatives in pattern search methods. SIAM J. Optim. 18, 537-555 (2007)

41. Custódio, A.L., Vicente, L.N.: SID-PSM: A Pattern Search Method Guided by Simplex Derivatives for Use in Derivative-Free Optimization. Departamento de Matemática, Universidade de Coimbra, Coimbra (2008)

42. Deming, S.N., Parker, L.R. Jr., Denton, M.B.: A review of simplex optimization in analytical chemistry. Crit. Rev. Anal. Chem. 7, 187-202 (1974)

43. Desai, R.: A Comparison of Algorithms for Optimizing the Omega Function. Master's thesis, Department of Chemical Engineering, Carnegie Mellon University, Pittsburgh (2010)

44. Eberhart, R.,Kennedy, J.: A new optimizer using particle swarm theory. In: Proceedings of the 6th International Symposium on Micro Machine and Human Science, Nagoya, pp. 39-43 (1995)

45. Eldred, M.S., Adams, B.M., Gay, D.M., Swiler, L.P., Haskell, K., Bohnhoff, W.J., Eddy, J.P., Hart, W.E., Watson, J-P, Hough, P.D., Kolda, T.G., Williams, P.J., Martinez-Canales, M.L., DAKOTA, A.: Multilevel Parallel Object-Oriented Framework for Design Optimization, Parameter Estimation, Uncertainty Quantification, and Sensitivity Analysis: Version 4.2 User's Manual. Sandia National Laboratories, Albuquerque (2008)

46. Fan, S.S., Zahara, E.: A hybrid simplex search and particle swarm optimization for unconstrained optimization. Eur. J. Oper. Res. 181, 527-548 (2007)

47. Finkel, D.E., Kelley, C.T.: Additive scaling and the DIRECT algorithm. J. Glob. Optim. 36, 597608 (2006)

48. Fowler, K.R., Reese, J.P., Kees, C.E., Dennis, J.E. Jr., Kelley, C.T., Miller, C.T., Audet, C., Booker, A.J., Couture, G., Darwin, R.W., Farthing, M.W., Finkel, D.E., Gablonsky, J.M., Gray, G., Kolda, T.G.: A comparison of derivative-free optimization methods for groundwater supply and hydraulic capture community problems. Adv. Water Resour. 31, 743-757 (2008)

49. Gablonsky, J.M.: Modifications of the DIRECT Algorithm. PhD thesis, Department of Mathematics, North Carolina State University, Raleigh (2001) 
50. Gilmore, P., Kelley, C.T.: An implicit filtering algorithm for optimization of functions with many local minima. SIAM J. Optim. 5, 269-285 (1995)

51. GLOBAL Library. http://www.gamsworld.org/global/globallib.htm

52. Gray, G., Kolda, T., Sale, K., Young, M.: Optimizing an empirical scoring function for transmembrane protein structure determination. INFORMS J. Comput. 16, 406-418 (2004)

53. Gutmann, H.-M.: A radial basis function method for global optimization. J. Glob. Optim. 19, 201227 (2001)

54. Han, J., Kokkolaras, M., Papalambros, P.Y.: Optimal design of hybrid fuel cell vehicles. J. Fuel Cell Sci. Technol. 5, 041014 (2008)

55. Hansen, N.: The CMA Evolution Strategy: A tutorial. http://www.lri.fr/hansen/cmaesintro.html

56. Hansen, N.: The CMA evolution strategy: a comparing review. In: Lozano, J.A., Larranaga, P., Inza, I., Bengoetxea, E. (eds.) Towards a New Evolutionary Computation. Advances on Estimation of Distribution Algorithms, pp. 75-102. Springer, Berlin (2006)

57. Hayes, R.E., Bertrand, F.H., Audet, C., Kolaczkowski, S.T.: Catalytic combustion kinetics: using a direct search algorithm to evaluate kinetic parameters from light-off curves. Can. J. Chem. Eng. 81, 11921199 (2003)

58. Holland, J.H.: Adaptation in Natural and Artificial Systems. The University of Michigan Press, Ann Arbor (1975)

59. Holmström, K.: Private Communication (2009)

60. Holmström, K., Göran, A.O., Edvall, M.M.: User's Guide for TOMLAB 7. Tomlab Optimization. http:// tomopt.com

61. Holmström, K., Göran, A.O., Edvall, M.M.: User's Guide for TOMLAB/CGO. Tomlab Optimization (2007). http://tomopt.com

62. Holmström, K., Göran, A.O., Edvall, M.M.: User's Guide for TOMLAB / OQNLP. Tomlab Optimization (2007). http://tomopt.com

63. Holmström, K., Quttineh, N.-H., Edvall, M.M.: An adaptive radial basis algorithm (ARBF) for expensive black-box mixed-integer constrained global optimization. Optim. Eng. 9, 311-339 (2008)

64. Hooke, R., Jeeves, T.A.: Direct search solution of numerical and statistical problems. J. Assoc. Comput. Mach. 8, 212-219 (1961)

65. Huyer, W., Neumaier, A.: Global optimization by multilevel coordinate search. J. Glob. Optim. 14, 331355 (1999)

66. Huyer, W., Neumaier, A.: SNOBFIT—Stable noisy optimization by branch and fit. ACM Trans. Math. Softw. 35, 1-25 (2008)

67. Hvattum, L.M., Glover, F.: Finding local optima of high-dimensional functions using direct search methods. Eur. J. Oper. Res. 195, 31-45 (2009)

68. Ingber, L.: Adaptive Simulated Annealing (ASA). http://www.ingber.com/\#ASA

69. Järvi, T.: A Random Search Optimizer with an Application to a Max-Min Problem. Technical report, Pulications of the Institute for Applied Mathematics, University of Turku (1973)

70. Jones, D.R.: A taxonomy of global optimization methods based on response surfaces. J. Glob. Optim. 21, 345-383 (2001)

71. Jones, D.R.: The DIRECT global optimization algorithm. In: Floudas, C.A., Pardalos, P.M. (eds.) Encyclopedia of Optimization, vol. 1, pp. 431-440. Kluwer, Boston (2001)

72. Jones, D.R., Perttunen, C.D., Stuckman, B.E.: Lipschitzian optimization without the Lipschitz constant. J. Optim. Theory Appl. 79, 157-181 (1993)

73. Jones, D.R., Schonlau, M., Welch, W.J.: Efficient global optimization of expensive black-box functions. J. Glob. Optim. 13, 455-492 (1998)

74. Kelley, C.T.: Users Guide for IMFIL version 1.0. http://www4.ncsu.edu/ctk/imfil.html

75. Kelley, C.T.: Detection and remediation of stagnation in the Nelder-Mead algorithm using a sufficient decrease condition. SIAM J. Optim. 10, 43-55 (1999)

76. Kelley, C.T.: Iterative Methods for Optimization. SIAM, Philadelphia (1999)

77. Kennedy, J., Eberhart, R.: Particle swarm optimization. In: Proceedings of the IEEE International Conference on Neural Networks, Piscataway, pp. 1942-1948 (1995)

78. Kirkpatrick, S., Gelatt, C.D., Vecchi, M.P.: Optimization by simulated annealing. Science 220, 671680 (1983)

79. Kolda, T.G., Lewis, R.M., Torczon, V.J.: Optimization by direct search: new perspectives on some classical and modern methods. SIAM Rev. 45, 385-482 (2003)

80. Kolda, T.G., Torczon, V.J.: On the convergence of asynchronous parallel pattern search. SIAM J. Optim. 14, 939-964 (2004)

81. Lagarias, J.C., Reeds, J.A., Wright, M.H., Wright, P.E.: Convergence properties of the Nelder-Mead simplex method in low dimensions. SIAM J. Optim. 9, 112-147 (1998) 
82. LeDigabel, S.: NOMAD User Guide Version 3.3. Technical report, Les Cahiers du GERAD (2009)

83. Lewis, R.M., Torczon, V.J.: Pattern search algorithms for bound constrained minimization. SIAM J. Optim. 9, 1082-1099 (1999)

84. Lewis, R.M., Torczon, V.J.: Pattern search algorithms for linearly constrained minimization. SIAM J. Optim. 10,917-941 (2000)

85. Lewis, R.M., Torczon, V.J.: A globally convergent augmented lagrangian pattern search algorithm for optimization with general constraints and simple bounds. SIAM J. Optim. 12, 1075-1089 (2002)

86. Liepins, G.E., Hilliard, M.R.: Genetic algorithms: foundations and applications. Ann. Oper. Res. 21, 31-58 (1989)

87. Lin, Y., Schrage, L.: The global solver in the LINDO API. Optim. Methods Softw. 24, 657-668 (2009)

88. Lucidi, S., Sciandrone, M.: On the global convergence of derivative-free methods for unconstrained minimization. SIAM J. Optim. 13, 97-116 (2002)

89. Lukšan, L., Vlček, J.: Test Problems for Nonsmooth Unconstrained and Linearly Constrained Optimization. Technical report, Institute of Computer Science, Academy of Sciences of the Czech Republic (2000). http://www3.cs.cas.cz/ics/reports/v798-00.ps

90. Marsden, A.L., Feinstein, J.A., Taylor, C.A.: A computational framework for derivative-free optimization of cardiovascular geometries. Comput. Methods Appl. Mech. Eng. 197, 1890-1905 (2008)

91. Marsden, A.L., Wang, M., Dennis, J.E. Jr., Moin, P.: Optimal aeroacustic shape design using the surrogate management framework. Optim. Eng. 5, 235-262 (2004)

92. Marsden, A.L., Wang, M., Dennis, J.E. Jr., Moin, P.: Trailing-edge noise reduction using derivative-free optimization and large-eddy simulation. J. Fluid Mech. 5, 235-262 (2007)

93. Matheron, G.: Principles of geostatistics. Econ. Geol. 58, 1246-1266 (1967)

94. McKinnon, K.I.M.: Convergence of the Nelder-Mead simplex method to a nonstationary point. SIAM J. Optim. 9, 148-158 (1998)

95. Metropolis, N., Rosenbluth, A.W., Rosenbluth, M.N., Teller, A.H., Teller, E.: Equation of state calculations by fast computing machines. J. Chem. Phys. 21, 1087-1092 (1953)

96. Mongeau, M., Karsenty, H., Rouzé, V., Hiriart-Urruty, J.B.: Comparison of public-domain software for black box global optimization. Optim. Methods Softw. 13, 203-226 (2000)

97. Moré, J., Wild, S.: Benchmarking derivative-free optimization algorithms. SIAM J. Optim. 20, 172-191 (2009)

98. Mugunthan, P., Shoemaker, C.A., Regis, R.G.: Comparison of function approximation, heuristic, and derivative-based methods for automatic calibration of computationally expensive groundwater bioremediation models. Water Resour. Res. 41, W11427 (2005)

99. Nelder, J.A., Mead, R.: A simplex method for function minimization. Comput. J. 7, 308-313 (1965)

100. Nesterov, Y.: Gradient methods for minimizing composite objective function. CORE Discussion Paper 2007/76 (2007)

101. Neumaier, A.: MCS: Global Optimization by Multilevel Coordinate Search. http://www.mat.univie.ac. at/neum/software/mcs/

102. Neumaier, A., Shcherbina, O., Huyer, W., Vinkó, T.: A comparison of complete global optimization solvers. Math. Program. 103, 335-356 (2005)

103. Oeuvray, R.: Trust-Region Methods Based on Radial Basis Functions with Application to Biomedical Imaging. PhD thesis, Institute of Mathematics, Swiss Federal Institute of Technology, Lausanne (2005, March)

104. Orosz, J.E., Jacobson, S.H.: Finite-time performance analysis of static simulated annealing algorithms. Comput. Optim. Appl. 21, 21-53 (2002)

105. Pintér, J.: Homepage of Pintér Consulting Services. http://www.pinterconsulting.com/

106. Pintér, J.D.: Global Optimization in Action: Continuous and Lipschitz Optimization. Algorithms, Implementations and Applications. Nonconvex Optimization and its Applications. Kluwer, Dordrecht (1995)

107. Pintér, J.D., Holmström, K., Göran, A.O., Edvall, M.M.: User's Guide for TOMLAB/LGO. Tomlab Optimization (2006). http://tomopt.com

108. Plantenga, T.D.: HOPSPACK 2.0 User Manual. Technical Report SAND2009-6265, Sandia National Laboratories, Albuquerque (2009)

109. Powell, M.J.D. A direct search optimization method that models the objective and constraint functions by linear interpolation. In: Gomez, S., Hennart, J-P. (eds.) Advances in Optimization and Numerical Analysis, pp. 51-67. Kluwer, Dordrecht (1994)

110. Powell, M.J.D.: A direct search optimization method that models the objective by quadratic interpolation. In: Presentation at the 5th Stockholm Optimization Days (1994)

111. Powell, M.J.D.: Recent Research at Cambridge on Radial Basis Functions. Technical report, Department of Applied Mathematics and Theoretical Physics, University of Cambridge (1998) 
112. Powell, M.J.D.: UOBYQA: unconstrained optimization by quadratic approximation. Math. Program. 92, 555-582 (2002)

113. Powell, M.J.D.: The NEWUOA software for unconstrained optimization without derivatives. In: Di Pillo, G., Roma, M. Large-Scale Nonlinear Optimization, pp. 255-297. Springer, New York (2006)

114. Powell, M.J.D.: Developments of NEWUOA for minimization without derivatives. IMA J. Numer. Anal. 28, 649-664 (2008)

115. Powell, M.J.D.: The BOBYQA Algorithm for Bound Constrained Optimization Without Derivatives. Technical report, Department of Applied Mathematics and Theoretical Physics, University of Cambridge (2009)

116. Princeton Library. http://www.gamsworld.org/performance/princetonlib/princetonlib.htm

117. Regis, R.G., Shoemaker, C.A.: Constrained global optimization of expensive black box functions using radial basis functions. J. Glob. Optim. 31, 153-171 (2005)

118. Regis, R.G., Shoemaker, C.A.: Improved strategies for radial basis function methods for global optimization. J. Glob. Optim. 37, 113-135 (2007)

119. Richtarik, P.: Improved algorithms for convex minimization in relative scale. SIAM J. Optim. (2010, to appear). http://www.optimization-online.org/DB_FILE/2009/02/2226.pdf

120. Rios, L.M.: Algorithms for Derivative-Free Optimization. PhD thesis, Department of Industrial and Enterprise Systems Engineering, University of Illinois, Urbana (2009, May)

121. Romeo, F., Sangiovanni-Vincentelli, A.: A theoretical framework for simulated annealing. Algorithmica 6, 302-345 (1991)

122. Sacks, J., Welch, W.J., Mitchell, T.J., Wynn, H.P.: Design and analysis of computer experiments. Stat. Sci. 4, 409-423 (1989)

123. Sahinidis, N.V., Tawarmalani, M.: BARON 7.5: Global Optimization of Mixed-Integer Nonlinear Programs, User's Manual (2005)

124. Sandia National Laboratories: The Coliny Project. https://software.sandia.gov/trac/acro/wiki/Overview/ Projects

125. Scheinberg, K.: Manual for Fortran Software Package DFO v2.0 (2003)

126. Schonlau, M.: Computer Experiments and Global Optimization. PhD thesis, Department of Statistics, University of Waterloo, Waterloo (1997)

127. Serafini, D.B.: A Framework for Managing Models in Nonlinear Optimization of Computationally Expensive Functions. PhD thesis, Department of Computational and Applied Mathematics, Rice University, Houston (1998, Nov)

128. Shah, S.B., Sahinidis, N.V.: SAS-Pro: Simultaneous residue assignment and structure superposition for protein structure alignment. PLoS ONE 7(5), e37493 (2012)

129. Shubert, B.O.: A sequential method seeking the global maximum of a function. SIAM J. Numer. Anal. 9, 379-388 (1972)

130. Smith, R.L.: Efficient Monte Carlo procedures for generating points uniformly distributed over bounded regions. Oper. Res. 32, 1296-1308 (1984)

131. Søndergaard, J.: Optimization Using Surrogate Models_-by the Space Mapping Technique. PhD thesis, Technical University of Denmark, Department of Mathematical Modelling, Lingby (2003)

132. Spendley, W., Hext, G.R., Himsworth, F.R.: Sequential application for simplex designs in optimisation and evolutionary operation. Technometrics 4, 441-461 (1962)

133. Tawarmalani, M., Sahinidis, N.V.: A polyhedral branch-and-cut approach to global optimization. Math. Program. 103, 225-249 (2005)

134. Torczon, V.J.: On the convergence of multidirectional search algorithms. SIAM J. Optim. 1, 123145 (1991)

135. Torczon, V.J.: On the convergence of pattern search algorithms. SIAM J. Optim. 7, 1-25 (1997)

136. Tseng, P.: Fortified-descent simplicial search method: a general approach. SIAM J. Optim. 10, 269288 (1999)

137. Vaz, A.I.F.: PSwarm Home Page. http://www.norg.uminho.pt/aivaz/pswarm/

138. Vaz, A.I.F., Vicente, L.N.: A particle swarm pattern search method for bound constrained global optimization. J. Glob. Optim. 39, 197-219 (2007)

139. Wang, H.: Application of Derivative-Free Algorithms in Powder Diffraction. Master's thesis, Department of Chemical Engineering, Carnegie Mellon University, Pittsburgh (2011)

140. Wild, S.M., Regis, R.G., Shoemaker, C.A.: ORBIT: Optimization by radial basis function interpolation in trust-regions. SIAM J. Sci. Comput. 30, 3197-3219 (2008)

141. Winfield, D.: Function and Functional Optimization by Interpolation in Data Tables. PhD thesis, Harvard University, Cambridge (1969) 
142. Winslow, T.A., Trew, R.J., Gilmore, P., Kelley, C.T.: Simulated performance optimization of gaas mesfet amplifiers. In: IEEE/Cornell Conference on Advanced Concepts in High Speed Semiconductor Devices and Circuits, Piscataway, pp. 393-402 (1991)

143. Zhao, Z., Meza, J.C., Van Hove, M.: Using pattern search methods for surface structure determination of nanomaterials. J. Phys. Condens. Matter 18, 8693-8706 (2006)

144. Zheng, Y.: Pairs Trading and Portfolio Optimization. Master's thesis, Department of Chemical Engineering, Carnegie Mellon University, Pittsburgh (2011) 